\title{
SOME NONCOMMUTATIVE MINIMAL SURFACES
}

\author{
D. ROGALSKI, S. J. SIERRA, AND J. T. STAFFORD
}

\begin{abstract}
In the ongoing programme to classify noncommutative projective surfaces (connected graded noetherian domains of Gelfand-Kirillov dimension three) a natural question is to determine the minimal models within any birational class.

In this paper we show that the generic noncommutative projective plane (corresponding to the three dimensional Sklyanin algebra) as well as noncommutative analogues of $\mathbb{P}^{1} \times \mathbb{P}^{1}$ and the more general Van den Bergh quadrics satisfy very strong minimality conditions. Translated into an algebraic question, where one is interested in a maximality condition, we prove the following result.
\end{abstract}

Theorem A: Let $R$ be a Sklyanin algebra or a Van den Bergh quadric that is infinite dimensional over its centre and let $A \supseteq R$ be any connected graded noetherian maximal order, with the same graded quotient ring as $R$. Then, up to taking Veronese rings, $A$ is isomorphic to $R$.

Let $T$ be an elliptic algebra (that is, the coordinate ring of a noncommutative surface containing an elliptic curve). Then, under an appropriate homological condition, we prove that every connected graded noetherian overring of $T$ is obtained by blowing down finitely many lines (line modules) of self-intersection $(-1)$.

\section{CONTENTS}

1. Introduction

2. Generalities

3. Some key lemmas

4. Overrings of locally simple elliptic algebras

5. Algebras with $A_{1}$ singularities

6. Sklyanin algebras and Van den Bergh quadrics

7. Overrings not contained in $T_{(g)}$

8. General overrings in the locally hereditary case

9. Gelfand-Kirillov dimension

Appendix A. Commutative algebras

Index of Notation

(1)

References

Date: April 27, 2020.

2010 Mathematics Subject Classification. Primary: 14A22, 16P40, 16S38, 16W50; Secondary: 14H52, 14E30.

Key words and phrases. Noncommutative projective geometry, Sklyanin algebras, noncommutative minimal models. The first author was partially supported by the NSF grant DMS-1201572 and the NSA grant H98230-15-1-0317.

The second author was partially supported by EPSRC grant EP/M008460/1. 


\section{INTRODUCTION}

The classification of noetherian, connected graded domains $R$ of Gelfand-Kirillov dimension 3 (or the corresponding noncommutative surfaces, written qgr- $R$ ) is one of the major open problems in noncommutative algebraic geometry. The classification has been solved in many particular cases and those solutions have led to some fundamental advances in the subject; see, for example, [ATV1, RSS2, KRS, SmV, VB2, VB3]. In $\underline{\mathrm{Ar}}$, Artin conjectured that, birationally at least, there is a short list of such surfaces. More precisely, the corresponding division rings of rational functions are either: (1) finite over their centre; (2) Ore extensions of $\mathbb{k}(C)$ for a curve $C$; or (3) the division ring associated to a Sklyanin algebra $S k l$, as defined in Example 6.1 Artin's conjecture is completely open, but this then leaves the question of classifying the algebras in each birational class. Case (1) was largely resolved in $\underline{\mathrm{KRS}}, \mathbf{R S}$, Si], while it is expected that case (2) will be answered by an amalgam of the methods developed cases (1) and (3). Thus a fundamental part of the classification problem, and the motivating question for this paper is:

What are the connected graded noetherian algebras $R$ that are birational to a Sklyanin algebra Skl?

In this paper, such algebras are always assumed to be infinite dimensional over their centres.

A natural approach to this problem is to follow the commutative classification of rational surfaces, which we briefly review. Here, one first classifies the minimal models: smooth projective surfaces $X$ with the property that any birational morphism from $X$ to a smooth projective surface $Y$ is an isomorphism [Sh, p. 175]. It is a consequence of Zariski's Main Theorem and Castelnuovo's contraction criterion that:

Theorem 1.1. A smooth projective surface $X$ is a minimal model if and only if $X$ contains no lines of self-intersection $(-1)$.

In fact much more is true.

Theorem 1.2. ([ $\mathrm{Ha}$, Corollary V.5.4]) If $X$ and $Y$ are smooth projective surfaces, then any birational morphism $X \rightarrow Y$ factors as a composition of finitely many monoidal transformations (contractions of lines of self-intersection $(-1))$.

Theorem 1.3. ([Ha, Theorem V.5.8, Remark V.5.8.4])

(1) Any smooth projective surface $X$ has a birational morphism to a minimal model.

(2) The minimal rational surfaces are known: they are $\mathbb{P}^{2}$ and Hirzebruch surfaces $\mathbb{F}_{n}$ for $n \neq 1$.

In this paper we move the classification programme for noncommutative surfaces forward by giving noncommutative analogues of Theorems 1.1 and Theorem 1.2 for algebras birational to $S k l$.

We note that noncommutative analogues of blowing up points are understood (see [VB2, Rg, RSS2]) and this has, for example, been used to classify the noetherian subalgebras of $S k l$ that are birational to that algebra $[\mathrm{RSS2}, \mathrm{BH}]$. Moreover, a noncommutative analogue of contraction or blowing down has been developed in RSS4, showing in particular that one really can contract lines (or more formally line modules) 
of self-intersection $(-1)$. Beyond that, what one might call the birational geometry of noncommutative projective surfaces is wide open. In particular, it is important to understand whether noncommutative minimal models exist and, indeed, how they should be defined. Whatever the definition, one would expect that the Sklyanin algebra $S k l$ and Van den Bergh's quadrics $Q_{V d B}$ are indeed examples (see Example 6.3 and [VB3] for the definition).

The main aim of this paper is to show these algebras are indeed minimal models; indeed they satisfy a minimality property that is far stronger than is possible in the commutative situation. This is provided by Theorem A from the abstract. The reason why this is described in terms of overrings will be explained below but we will continue to use the geometric notation since we feel this gives the better intuition for the results proved here.

To formalise these statements we need some definitions. In the paper, all rings will be algebras over a fixed algebraically closed field $\mathbb{k}$, while in the introduction we also assume that $\mathbb{k}$ has characteristic zero. A $\mathbb{k}$-algebra $R$ is connected graded or $c g$ if $R=\bigoplus_{n \geqslant 0} R_{n}$ is a finitely generated $\mathbb{N}$-graded algebra with $R_{0}=\mathbb{k}$. For such a ring $R$, the category of graded noetherian right $R$-modules will be denoted gr- $R$ with quotient category qgr- $R$ obtained by quotienting out the Serre subcategory of finite dimensional modules. An effective intuition is to regard qgr- $R$ as the category of coherent sheaves on the (nonexistent) space $\operatorname{Proj}(R)$. The graded quotient ring $Q_{g r}(R)$ of $R$ is obtained by inverting the non-zero homogeneous elements and two such domains $R$ and $S$ are birational if $Q_{g r}(R)_{0} \cong Q_{g r}(S)_{0}$.

As is explained in Rg, RSS2, when one works with algebras related to $S k l$ it is convenient to work with the following general class of algebras. An elliptic algebra is a cg domain $T$ containing a distinguished central element $g \in T_{1}$ so that $T / g T$ is isomorphic to a twisted homogeneous coordinate ring $B=B(E, \mathcal{N}, \tau)$, where $E$ is an elliptic curve equipped with an ample invertible sheaf $\mathcal{N}$ (which we assume in the introduction to have degree $\geqslant 3$ ) and an infinite order automorphism $\tau$. See Section 2 for more details. For example, the Veronese rings $T=S k l^{(3)}$ and $T=Q_{V d B}{ }^{(2)}$ are elliptic; the Veronese ring is needed to ensure that the central element has degree one, but this is a fairly harmless change since qgr- $S \cong$ qgr- $T$ for $S=S k l$, respectively $Q_{V d B}$. By Lemma A.1, a ring that is commutative but satisfies all other properties of an elliptic algebra is the anticanonical coordinate ring of a del Pezzo surface, so this is a natural class of algebras to consider.

The ring-theoretic notion of blowing up a ring $R$ in $\mathrm{Rg}$ produces a subring $\widetilde{R} \subseteq R$, although as its construction is not used in this paper we will not repeat the definition. As noted in [Rg, Introduction], this notion of blowing up is equivalent to Van den Bergh's categorical notion VB2. Subalgebras described in terms of blowups also appear naturally in the commutative case (see Remark A.4 for an example).

The appropriate notion of blowing down for an elliptic algebra $T$ has been studied in RSS4]. In brief, define a cyclic graded right $T$-module $L$ to be a line module if has the Hilbert series $(1-t)^{-2}$ of $\mathbb{k}[x, y]$. Then one can contract (blow down) any line module $L$ satisfying the noncommutative notion of self-intersection

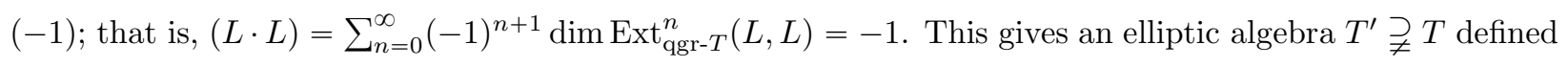
by the property that $T^{\prime} / T$ is a suitable direct sum of shifts of copies of $L$. Crucially, $T^{\prime}$ has a point module 
can be blown up to recover $T$. Conversely, blowing up a point on $T$ constructs a subalgebra $\widetilde{T}$ with a line module $L$ that can be contracted to recover $T$. See [RSS4, Introduction] for the results mentioned here.

So, in the noncommutative context, what is the appropriate definition of a minimal model? Since contracting a curve corresponds to taking a certain overring within the graded quotient ring, any noncommutative analogue of a minimal surface should be an algebra with few overrings. However, beyond that, it is unclear how best to translate the commutative definition. Fortunately, it does not matter since the elliptic algebras of interest satisfy a very strong maximality property, which we can take as our definition.

Definition 1.4. Given an elliptic algebra $T$, with its central element $g \in T_{1}$, write $T_{(g)}$ for the localisation obtained by inverting the homogeneous elements from $T \backslash g T$. Then a minimal (noncommutative) elliptic surface is an elliptic algebra $T$ which has the property that, if $R$ is a cg noetherian algebra with $T \subseteq R \subset T_{(g)}$, then $T=R$.

As will be seen in Theorem 1.8, the restriction to subrings of $T_{(g)}$ is also unnecessary.

Definition 1.4 is so strong that one may wonder if there are any minimal elliptic surfaces at all. After all, every cg commutative noetherian domain $A$ of Krull dimension $\geqslant 2$ has a proper cg noetherian overringsimply adjoin an element of positive degree from its graded quotient ring. In contrast, in the noncommutative setting minimal elliptic surfaces do exist, as our main theorem shows.

Theorem 1.5. (See Theorem 6.16) Let $T=Q_{V d B}{ }^{(2)}$ be the second Veronese of a Van den Bergh quadric or $T=S k l^{(3)}$, the third Veronese of a quadratic Sklyanin algebra Skl. Then $T$ is a minimal elliptic surface.

Theorem 1.5 is a consequence of the following noncommutative version of Theorem 1.1 which further justifies our definition of a minimal model.

Theorem 1.6. (See Theorems 5.5 and 8.12) Let $T$ be an elliptic algebra with no line modules of selfintersection $(-1)$. Assume that $\left(T\left[g^{-1}\right]\right)_{0}$ is hereditary. Then $T$ is a minimal elliptic surface.

The conditions of this theorem always hold for $T=S k l^{(3)}$ and hold generically for $T=Q_{V d B}{ }^{(2)}$.

We note that Theorem 1.5 easily lifts to give the analogous result for the original rings $S k l$ and $Q_{V d B}$.

Corollary 1.7. (See Corollary 6.18) Set $S=Q_{V d B}$ or $S=S k l$, with corresponding central element $g$. If $S \subseteq U \subset S_{(g)}$ for some cg noetherian ring $U$ then $S=U$.

The focus on the localised ring $T_{(g)}$ in Definition 1.4 may seem mysterious. However, as we next discuss, minimal models still have few overrings without this restriction. Let $T$ be one of the algebras from Theorem 1.5, or indeed any minimal elliptic surface, with its central element $g \in T_{1}$. Then there always exist cg noetherian overrings of $T$ with the same graded quotient ring. Indeed, for any integer $n \geqslant 2$ one has $T \varsubsetneqq \mathbb{k}\left\langle T_{n} g^{1-n}\right\rangle$. This is of course a rather "cheap" counterexample since after a change of grading, $\mathbb{k}\left\langle T_{n} g^{1-n}\right\rangle \cong T^{(n)}$ under the homomorphism $x g^{1-n} \mapsto x$ for $x \in T_{n}$. As we will see, rings like this are essentially the only other cg noetherian overrings of $T$. 
In the next result, a $\mathbb{Z}$-graded Goldie domain $U$ is a maximal order if it satisfies the following condition: If $V$ is a $\mathbb{Z}$-graded $\mathbb{k}$-algebra with $U \subseteq V \subset Q_{g r}(U)$ that satisfies $a V b \subseteq U$ for some $a, b \in U \backslash\{0\}$, then $V=U$. This condition is equivalent to its ungraded analogue and is the appropriate noncommutative analogue of an integrally closed domain. We note that elliptic algebras are maximal orders (see Remark 6.8).

Theorem 1.8. (See Theorem [7.1) Let $T$ be any minimal elliptic surface, with central element $g \in T_{1}$, and let $R$ be a cg noetherian $\mathbb{k}$-algebra with $T \subseteq R \subset Q_{g r}(T)$. If $R$ is a maximal order, then $R^{(m)} \cong T^{(n)}$ for some integers $m, n \geqslant 1$. The analogous theorem also holds for the rings $S k l$ and $Q_{V d B}$.

In the commutative case, Theorem 1.2 follows from the Néron-Severi theorem and Castelnuovo's contraction criterion. In Section 8 we use the techniques from the proof of Theorem [1.5, together with the noncommutative version of Castelnuovo's contraction criterion from RSS4, to give a noncommutative analogue of this result. Given a graded $T$-module $M$ over an elliptic algebra $T$, we define $T^{\circ}=T\left[g^{-1}\right]_{0}$ and $M^{\circ}=M \otimes_{T} T\left[g^{-1}\right]_{0}$.

Theorem 1.9. (See Corollary 8.3,) Let $T$ be an elliptic algebra for which $T^{\circ}$ is hereditary and let $T \subseteq R \subset$ $T_{(g)}$ be any noetherian cg overring. Then there is a module-finite ring extension $R \subseteq R^{\prime}$ such that $R^{\prime}$ is obtained from $T$ by contracting finitely many line modules $L$ of self-intersection -1 .

We remark that finitely generated but non-noetherian cg overrings of minimal elliptic surfaces $T$ obviously exist (just take the ring generated by $T$ and a homogeneous element of positive degree from $\left.T_{(g)}\right)$. However, the resulting ring has many unpleasant properties (see, for example, Proposition 4.8 and Corollary [5.7). Moreover (except for trivial overrings like $R=\mathbb{k}\left\langle T_{n} g^{1-n}\right\rangle$ ), the Gelfand-Kirillov dimension GKdim $R$ must jump; for example, if $R$ is a cg noetherian ring with $T \varsubsetneqq R \subset T_{(g)}$, then $\operatorname{GKdim} R \geqslant 4>3=\operatorname{GKdim} T$ (see Section 9 and in particular Corollary 9.7).

The idea of the proof of Theorem 1.5 is as follows. The starting point is that, by Lemma 6.14 $T$ has no line modules to contract. But suppose that $T$ does have a noetherian cg overring $T \varsubsetneqq R \subset T_{(g)}$. Form the localisation $R^{\circ}:=R\left[g^{-1}\right]_{0} \supsetneqq T^{\circ}:=T\left[g^{-1}\right]_{0}$. In the cases of interest, one can always reduce to the case when $T^{\circ}$ is simple and is either hereditary or has a mild singularity. For simplicity assume that $T^{\circ}$ is hereditary; the argument is easiest to explain here but does still give the general idea. Also, after possibly replacing $R$ by a harmless, finitely generated extension one can assume that $R=\Phi\left(R^{\circ}\right)=\bigoplus_{n \in \mathbb{Z}}\left\{a \in\left(T_{(g)}\right)_{n}: a g^{-n} \in R^{\circ}\right\}$ (see Lemma 3.9).

Now pick a simple submodule $\mathcal{N}=\mathcal{M} / T^{\circ} \subseteq R^{\circ} / T^{\circ}$. One can show that $\mathcal{M}=M^{\circ}$ for some module $M \supseteq T$ such that $N=M / T$ is a 2 -critical $T$-module with $N^{\circ} \cong \mathcal{N}$. Since $N$ cannot be a line module, one proves that there are many copies of submodules of $N$ inside $Q_{g r}(T) / T$, one of which, say $N^{\prime}=M^{\prime} / T$, is not contained in $R / T$. However, one still has $\left(N^{\prime}\right)^{\circ} \cong \mathcal{N} \cong N^{\circ}$. Now, as $T^{\circ}$ is hereditary, a classic result of Goodearl [Go] shows that $R^{\circ}$ will be a torsion-theoretic localisation of $T^{\circ}$. This means that $R^{\circ} / T^{\circ}$ will contain all possible copies of $N^{\circ}$ and in particular $\left(N^{\prime}\right)^{\circ}$. In other words, $\left(M^{\prime}\right)^{\circ} \subseteq R^{\circ}$ and hence $M^{\prime} \subseteq \Phi\left(R^{\circ}\right)=R$, giving the 
required contradiction. See Section 4 for the details. This argument is modified to work for algebras with $A_{1}$ singularities in Section 5 and, as is shown in Section 6 this in turn is enough to prove the theorem.

Finally, we note that noncommutative versions of Theorem 1.3 have yet to be established and are the subject of ongoing work. We believe that the full list of minimal models for the quantum rational case will consist of qgr- $R$ for $R=S k l$ and $R=Q_{V d B}$, as above, together with their Veronese rings and certain endomorphism algebras; see Remark 7.9. We conjecture that Theorems 1.5 and 1.9 can be extended to prove the ultimate goal that, given an elliptic algebra $R$, then one can obtain a minimal model $\widehat{R}$ from $R$ by contracting finitely many line modules. This in turn will prove much of Artin's programme for algebras birational to the Sklyanin algebra $S k l$. See Conjecture 8.13 and Remark 7.9 for more details.

\section{Generalities}

In this short section, we set up some of the basic notation and results used later in the paper. Fix an algebraically closed base field $\mathbb{k}$ of arbitrary characteristic. Let $T$ be an elliptic algebra with graded quotient ring $Q_{g r}(T) \cong D\left[g, g^{-1}\right]$, for the appropriate division $\operatorname{ring} D$. Thus $T / g T \cong B:=B(E, \mathcal{M}, \tau)$ is a twisted homogeneous coordinate ring, or TCR, over the elliptic curve $E$ and $\tau$ is an automorphism of $E$ of infinite order (and so $\tau$ is given by translation by a point of $E$ under the group law). Here $B=\oplus B_{n}$, where $B_{n}=H^{0}\left(E, \mathcal{M}_{n}\right)$ and $\mathcal{M}_{n}=\mathcal{M} \otimes \cdots \otimes \mathcal{M}^{\tau^{n-1}}$, with the natural multiplication. We say that $E$ is the elliptic curve associated to $T$ (or $B$ ) and define the degree of $T$ to be the degree of the line bundle $\mathcal{M}$. Unless stated otherwise, we assume in the body of the paper that $\operatorname{deg} \mathcal{M} \geqslant 2$ so that $B$ and $T$ are generated in degree 1 (see, for example, Rg, Lemma 3.1(2)]).

Definition 2.1. First, it is convenient to weaken the concept of a cg algebra. Define a $\mathbb{Z}$-graded $\mathbb{k}$-algebra $R$ to be finitely graded if $\operatorname{dim}_{\mathbb{k}} R_{n}<\infty$ for all $n$ and $R_{n} \neq 0$ for some $n>0$ (the final condition is included since it is convenient to exclude rings graded by $-\mathbb{N}$ ). Obviously, apart from $R=k$ itself, cg algebras, as defined in the introduction, are finitely graded.

Remark 2.2. The following observations will be used several times, usually without further comment.

(1) Suppose that $R$ is a $\mathbb{Z}$-graded domain with $\operatorname{dim}_{\mathbb{k}} R_{n} \geqslant 2$ for some $n \geqslant 0$ (as is always the case in this paper). If $R$ is not $\mathbb{N}$-graded, say with $R_{-a} \neq 0$, then $\left(R_{-a}\right)^{n}\left(R_{n}\right)^{a}$ contains an element $\alpha \in R_{0} \backslash \mathbb{k}$ and so $R_{0}$ contains the polynomial ring $\mathbb{k}[\alpha]$. Thus $R$ is not finitely graded. Equivalently, if $R$ is finitely graded then $R$ is necessarily $\mathbb{N}$-graded with $R_{0}=\mathbb{k}$.

(2) In a similar vein, if $R$ is a noetherian $\mathbb{N}$-graded $\mathbb{k}$-algebra with $R_{0}=\mathbb{k}$, then generators of the $R$-module $R \geqslant 1$ also generate $R$ as an algebra. Thus $R$ is cg.

We next review some important homological conditions. Throughout $\operatorname{Hom}_{R}(M, N)$ and $\operatorname{Ext}_{R}^{i}(M, N)$ will denote the given groups in the category of $R$-modules. When $M$ and $N$ are finitely generated $\mathbb{Z}$-graded modules over an $\mathbb{N}$-graded ring $R$, these carry a natural $\mathbb{Z}$-gradation. 
Definition 2.3. Let $A$ be a ring with $\operatorname{inj} \operatorname{dim}(A)<\infty$, in the sense that $A$ has finite injective dimension on both left and right. For a finitely generated right $A$-module $M$, write $j(M)=\min \left\{r: \operatorname{Ext}_{A}^{r}(M, A) \neq 0\right\}$ for the homological grade of $M$. Then $M$ is then called Cohen-Macaulay (or CM), if $\operatorname{Ext}_{A}^{j}(M, A)=0$ for all $j \neq j(M)$. The module $M$ is maximal Cohen-Macaulay (or MCM) if it is CM with $j(M)=0$.

A ring $A$ with $\operatorname{inj\operatorname {dim}}(A)<\infty$ is called Auslander-Gorenstein if the following holds: if $0 \leqslant p<q$ and $M$ is a finitely generated $A$-module, then $\operatorname{Ext}_{A}^{p}(N, A)=0$ for every submodule $N$ of $\operatorname{Ext}_{A}^{q}(M, A)$. Write $\operatorname{GKdim}(M)$ for the Gelfand-Kirillov dimension of an $A$-module $M$, as in [KL]. Let $A$ be an noetherian Auslander-Gorenstein $\mathbb{k}$-algebra with $\operatorname{GKdim}(A)<\infty$. The algebra $A$ is called Cohen-Macaulay (or CM), provided that $j(M)+\operatorname{GKdim}(M)=\operatorname{GKdim}(A)$ holds for every finitely generated $A$-module $M$. Note that an elliptic algebra is always Auslander-Gorenstein and CM by [RSS3, Proposition 2.4].

Let $M=\bigoplus_{n \in \mathbb{Z}} M_{n}$ be a non-zero $\mathbb{Z}$-graded module over a cg ring $R$ of finite Gelfand-Kirillov dimension such that $\operatorname{dim}_{\mathbb{k}} M_{n}<\infty$ for all $n$. Then $M$ is called $d$-critical if $\operatorname{GKdim} M=d \operatorname{but} \operatorname{GKdim}(M / N)<d$ for all non-zero submodules $N \subseteq M$. In order to avoid repetition, we always assume that critical $T$-modules are both finitely generated and graded. Similarly, $M$ is $d$-pure if $\operatorname{GKdim} M=\operatorname{GKdim} N=d$ for all non-zero submodules $N \subseteq M$. Note that, by [KL, Theorem 6.14], a $d$-critical $\mathbb{Z}$-graded module is automatically $d$-pure.

The Hilbert series of $M$ is defined to be hilb $M=\bigoplus_{n \in \mathbb{Z}}\left(\operatorname{dim}_{\mathbb{k}} M_{n}\right) t^{n}$. Finally, $M$ is a linear module if $M=M_{0} R$, with hilb $(M)=(1-t)^{-p}$ for some $p>0$. When $p=1$, respectively 2 , the module $M$ is called $a$ point module, respectively a line module. If $R$ is also an elliptic algebra, then $M$ is called torsion or Goldie torsion if, for all $m \in M$ there exists $t \in R \backslash\{0\}$ with $m t=0$. We say that $M$ is $g$-torsion if for all $m \in M$ one has $m g^{n}=0$ for some $n \geqslant 0$. The terms Goldie torsionfree and $g$-torsionfree then have their expected meaning. If $M$ is a graded $R$-module and $n \in \mathbb{Z}$, define $M[n]=\bigoplus_{j \in \mathbb{Z}} M[n]_{j}$, where $M[n]_{j}=M_{j+n}$.

Lemma 2.4. Let $B=B(E, \mathcal{L}, \tau)$ be a TCR over an elliptic curve $E$ with $\operatorname{deg} \mathcal{L} \geqslant 2$ and $|\tau|=\infty$.

(1) $B$ is Auslander-Gorenstein, $C M$ and generated by $B_{1}$ as an algebra.

(2) We have $\operatorname{Ext}_{B}^{i}(\mathbb{k}, B)=\delta_{i, 2} \mathbb{k}$.

(3) Let $M$ be a right (left) point module over $B$. Then $M$ is $C M$ and $\operatorname{Ext}_{B}^{1}(M, B)[1]$ is a left (right) point module.

Proof. For Part (1), see [RSS4, Lemma 2.2], for Part (2) see [Le, Theorem 6.3] and for Part (3) see [RSS4, Lemma 3.3] (this final result assumes that $\operatorname{deg} \mathcal{L} \geqslant 3$ but the proof also works when $\operatorname{deg} \mathcal{L}=2$ ).

Lemma 2.5. Let $T$ be an elliptic algebra. Let $M$ be a 2-pure finitely generated graded $g$-torsionfree $T$ module such that $M / M g$ is 1-pure. Then $M / M g$ has a filtration with shifted point module sub-factors $\left\{P\left(p_{i}\right)\left[m_{i}\right]: 1 \leqslant i \leqslant d\right\}$. Moreover, $\operatorname{Ext}_{T}^{1}(M, T)$ has Hilbert series $\sum_{i=1}^{d} s^{m_{i}+1} /(1-s)^{2}$.

Remark 2.6. In the lemma above, we write $P(p)$ for the $T / T g$-point module parameterised by $p \in E$; we shall not need the details of this parameterisation. The number $d$ is written $d=d(M)$. 
Proof. Since $M / M g$ is 1-pure, it has a composition series $0=N_{0} \subseteq N_{1} \subseteq \cdots \subseteq N_{d}=M$ with 1-critical factors $N_{i} / N_{i-1}$. By [RSS3, Lemma 2.8], the 1-critical modules over $B=T / g T$ are just shifted point modules, which proves the first statement. The second statement then follows from [RSS4, Lemma 5.4(2)] (once again, that result assumes that $\operatorname{deg} \mathcal{L} \geqslant 3$, but the proof also works when $\operatorname{deg} \mathcal{L}=2$ ).

Notation 2.7. Given a right module $M$ over an elliptic algebra $T$, set $E^{11}(M):=\operatorname{Ext}_{T}^{1}\left(\operatorname{Ext}_{T}^{1}(M, T), T\right)$. Recall that $T^{\circ}:=T\left[g^{-1}\right]_{0}=\bigcup_{n \geqslant 0} T_{n} g^{-n}$ and that $M^{\circ}:=M\left[g^{-1}\right]_{0} \cong\left(M \otimes_{T} T\left[g^{-1}\right]\right)_{0}$.

Lemma 2.8. Let $M$ be a 2-critical, g-torsionfree right module over an elliptic algebra $T$. Then

(1) $E^{11}(M)$ is the maximal essential extension of $M$ by finite-dimensional modules. Hence $E^{11}(M)$ is still $g$-torsionfree and 2-critical, with $\left(E^{11}(M)\right)^{\circ}=M^{\circ}$ and $\operatorname{Ext}_{T}^{1}\left(\mathbb{k}, E^{11}(M)\right)=0$;

(2) $E^{11}(M)$ is CM. Moreover $E^{11}(M) / E^{11}(M) g$ is 1-pure.

Proof. (1) The first statement follows, for example, from [Le, (4.6.6) and Remark 5.8(4)]. As such, $E^{11}(M) / M$ is annihilated by a power of $g$ and so $E^{11}(M)^{\circ}=M^{\circ}$. The other two assertions then follow easily.

(2) If $E^{11}(M) / E^{11}(M) g$ has a non-zero, finite dimensional submodule, say $W / E^{11}(M) g$, then $E^{11}(M) \cong$ $E^{11}(M) g[1]$ has a nontrivial extension by that finite-dimensional module, contradicting Part (1). Thus $E^{11}(M) / E^{11}(M) g$ is 1-pure. The CM condition then follows from Lemma 2.5 and [RSS4, Lemma 5.4].

\section{SOME KEY LEMMAS}

In this section we provide three technical lemmas that, nevertheless, lie at the heart of the proofs of the main theorems.

The first lemma is at the heart of the proof of Theorem 1.8, it shows that the structure of certain modules over an elliptic algebra $T$ can be perturbed without affecting their image in the localised category mod- $T^{\circ}$. This is used in Proposition 3.2 to get useful pertubations of $T$ modules. The final result of the section investigates the relationship between a cg algebra and its $g$-divisible hull, as defined below, which will be important in understanding general rings.

Lemma 3.1. Let $T$ be an elliptic algebra with a 2-critical, $g$-torsionfree $T$-module $M$ such that $M=E^{11}(M)$ and $\min \left\{i: M_{i} \neq 0\right\}=0$. Suppose that $M / M g$ has a filtration

$$
0=N(0) / M g \varsubsetneqq N(1) / M g \varsubsetneqq \cdots \varsubsetneqq N(d) / M g=M / M g
$$

with factors being (unshifted) point modules $\left\{P\left(p_{i}\right):=N(i) / N(i-1): 1 \leqslant i \leqslant d\right\}$. Then the following hold.

(1) $N:=N(1)$ is again 2-critical CM g-torsionfree, with $N^{\circ}=M^{\circ}$ and $\min \left\{i: N_{i} \neq 0\right\}=0$.

(2) However, now $N / N g$ has a filtration by shifted point modules $\left\{P\left(q_{i}\right)\left[n_{i}\right]: 1 \leqslant i \leqslant d\right\}$ where $n_{d}=0$ but $n_{i}=-1$ for $1 \leqslant i \leqslant d-1$.

Proof. (1) We may assume that $d>1$, as the result is trivial otherwise. The module $N$ is trivially 2-critical and $g$-torsionfree and hence also 2-pure. By Lemma 2.8 $E^{11}(N)$ is the largest essential extension of $N$ by 
finite-dimensional modules, while $M$ has no nontrivial such extensions. Thus, using the inclusion $E^{11}(N) \subseteq$ $I(N) \subseteq I(M)$, inside the injective hull $I(M)$, we conclude that $E^{11}(N) \subseteq M$. But, by construction, $M / N$ is filtered by shifted point modules and hence is 1-pure. Thus $E^{11}(N)=N$. Therefore, by Lemma $2.8, N$ is CM. Since $M \supseteq N \supseteq M g$, certainly $M^{\circ}=N^{\circ}$. Since $(N / M g)_{0}=P\left(p_{1}\right)_{0} \neq 0$, certainly $N_{0} \neq 0$ and hence $\min \left\{i: N_{i} \neq 0\right\}=0$.

(2) It remains to understand the shifted point module filtration of $N / N g$. Recall that $\operatorname{Tor}_{1}^{T}(L, T / T g)[1] \cong$ $\{x \in L: x g=0\}$ for any graded $T$-module $L$ (see, for example, $\mathrm{Rg}$, Equation (8.1)]). In particular, $\operatorname{Tor}_{1}^{T}(M, T / T g)=0$. Thus the short exact sequence $0 \rightarrow N \rightarrow M \rightarrow M / N \rightarrow 0$ induces an exact sequence

$$
0 \longrightarrow \operatorname{Tor}_{1}^{T}(M / N, T / T g) \longrightarrow N / N g \longrightarrow M / M g \longrightarrow M /(N+M g) \longrightarrow 0 .
$$

By [Rg, Equation (8.1)], again, $X:=\operatorname{Tor}_{1}^{T}(M / N, T / T g) \cong(M / N)[-1]$. Thus, by the definition of $N, X$ is filtered by the point modules $\left\{P\left(p_{i}\right)[-1]: 2 \leqslant i \leqslant d\right\}$, while $(N / N g) / X \cong(N+M g) / M g=N / M g$ is the point module $P\left(p_{1}\right)$. This defines the desired filtration of $N / N g$.

We now provide a useful application of Lemma 3.1

Proposition 3.2. Let $T$ be an elliptic algebra, with a finitely graded overring $T \varsubsetneqq R \subset T_{(g)}$. Assume that $T^{\circ}$ is simple and fix a simple $T^{\circ}$-submodule $\mathcal{S}$ of $R^{\circ} / T^{\circ}$. Then there is a 2-critical $g$-torsionfree $T$-module $M$ with $\min \left\{i: M_{i} \neq 0\right\}=0$ such that $M^{\circ}=\mathcal{S}$. Moreover, in the notation of Lemma 2.5 either

(1) $d(M)=1$ and $M$ is a line module, or

(2) $d(M)>1$ in which case there exists a module extension $T \varsubsetneqq L \subset T_{(g)}$ such that $L^{\circ} / T^{\circ} \cong \mathcal{S}$ but $L_{r} \supsetneqq T_{r}$ for some $r \leqslant 0$.

Remark 3.3. The significance of this result is that, for the algebras $T$ of interest, we will show that $L$ can also be embedded into the given overring $R$. This contradicts Remark 2.2(1). Thus $T$ must have line modules which, in turn, proves much of Theorem 1.6 .

Proof. Write $\mathcal{S} \cong T^{\circ} / \mathcal{J}$ for some right ideal $\mathcal{J}$ and set $I=\bigoplus_{n \in \mathbb{N}}\left\{x \in T_{n}: x g^{-n} \in \mathcal{J}\right\}$. Thus $I$ is a graded right ideal of $T$ such that $I^{\circ}=\mathcal{J}$ and so $(T / I)^{\circ} \cong \mathcal{S}$. By construction, $M=T / I$ is $g$-torsionfree. If $\operatorname{GKdim}(M)=1$ then, by the proof of [ATV2, Proposition 7.5], $\mathcal{S}=M^{\circ}$ would be finite-dimensional, contradicting the simplicity of $T^{\circ}$. Thus $\operatorname{GKdim}(M)=2$. Indeed, we claim that $M$ is 2-critical. To see this, suppose that $M$ has a proper factor $T / J$ with $\operatorname{GKdim}(T / J)=2$; we may assume that $T / J$ is 2 -critical. Then, by the definition of $I$, this forces $J^{\circ}=T^{\circ}$ and hence $J \supseteq g^{n} T$ for some $n$. By [ATV2, Proposition 2.36(vi)], $T / J$ has a prime annihilator and hence $J \supseteq g T$. Since $B=T / g T$ is 2-critical, this implies that $g T=J \supset I$. This is impossible by the definition of $I$ and implies that $M$ is 2-critical.

By Lemma 2.8, it is harmless to replace $M$ by $E^{11}(M)$ and so $M$ now has the properties described by that lemma. Note that $M g \cong M[-1]$ and so $M^{\circ} \cong M[n]^{\circ}$ for all $n \in \mathbb{Z}$. Thus we may also replace $M$ by some shift $M[n]$ and assume that $\min \left\{i: M_{i} \neq 0\right\}=0$. 
By Lemma 2.5 $M / M g$ has a filtration with $d=d(M)$ shifted point module subfactors. If $d=1$, then $M / M g$ is a shifted point module, and so $M$ has the Hilbert series $s^{m} /(1-s)^{2}$ of a shifted line module. Since $\min \left\{i: M_{i} \neq 0\right\}=0$, actually $M$ has Hilbert series $1 /(1-s)^{2}$. Since $M / M g$ is cyclic, so is $M$ and hence $M$ is a line module.

So suppose that $d \geqslant 2$. Let the point modules in the filtration of $M / M g$ be $\left\{P\left(p_{i}\right)\left[m_{i}\right]: 1 \leqslant i \leqslant d\right\}$. Since $\min \left\{i:(M / M g)_{i} \neq 0\right\}=0$, necessarily $m_{i}=0$ for some $i$. It may be that $m_{j}<0$ for some $j$, which is fine. If this is not the case, then $m_{i}=0$ for all $1 \leqslant i \leqslant d$. In this case, we can, by Lemma 3.1, replace $M$ by a second module with all of the properties of $M$ except that now $m_{d}=0$ and $m_{i}<0$ for $1 \leqslant i \leqslant d-1$.

By Lemma 2.5, $\operatorname{Ext}_{T}^{1}(M, T)$ has Hilbert series $\sum_{i=1}^{d} s^{m_{i}+1} /(1-s)^{2}$. By construction, some $m_{i}<0$ and so writing this Hilbert series as $\sum_{n \in \mathbb{Z}} c_{n} s^{n}$, we have $c_{n}>0$ for some $n \leqslant 0$. Fixing such an $n$, there therefore exists $0 \neq \theta \in \operatorname{Ext}_{T}^{1}(M, T)_{n}=\operatorname{Ext}_{T}^{1}(M, T[n])_{0}=\operatorname{Ext}_{T}^{1}(M[-n], T)_{0}$. Then $\theta$ corresponds to a (necessarily nonsplit) graded exact sequence $0 \rightarrow T \rightarrow X \rightarrow M[-n] \rightarrow 0$ for some module $X$. Now $T$ is AuslanderGorenstein and CM by $\mathrm{Rg}$, Theorem 6.3] and so $\operatorname{Ext}_{T}^{1}(N, T)=0$ for any module $N$ with $\operatorname{GKdim}(N) \leqslant 1$. Since $M[-n]$ is 2-critical, it follows easily that the extension $T \hookrightarrow X$ is essential. Thus we may embed $T \varsubsetneqq X \varsubsetneqq Q_{g r}(T)$, the graded quotient ring of $T$. Since $M[-n]$ is $g$-torsionfree, but (Goldie) torsion, it follows that, for any $x \in X$ there exists $t \in T \backslash g T$ such that $x t \in T$. In other words, $X \subseteq T_{(g)}$.

Finally, $X / T \cong M[-n]$, and since $M^{\circ} \cong \mathcal{S}$, one also has $M[-n]^{\circ} \cong \mathcal{S}$. However, $(X / T)_{n}=M[-n]_{n}=$ $M_{0} \neq 0$ with $n \leqslant 0$. Thus $L=X$ satisfies the conclusions of the proposition.

The main results of this paper will also cover non-elliptic algebras and we end the section with some technical results needed for this more general case.

Lemma 3.4. Suppose that $A$ is a $\mathrm{cg} \mathbb{k}$-algebra that is a domain with $\mathrm{GKdim} A=2$ and graded quotient ring $Q_{g r}(A)$. Assume that $Q_{g r}(A) \hookrightarrow G:=k(E)\left[t, t^{-1} ; \tau\right]$ with $|\tau|=\infty$.

Let $\{Q(i): i \geqslant 0\}$ be an ascending chain of graded $A$-sub-bimodules of $G$ that are finitely generated as both left and right $A$-modules. Then the chain is eventually stationary.

Proof. It does no harm to replace $A$ by some Veronese $\operatorname{ring} A^{(n)}$, and thereby assume that $A=A^{(n)}$, with $\operatorname{dim}_{\mathbb{k}} A_{n} \geqslant 2$. We emphasise that, here, we do not change the grading on $A$, since we cannot replace $Q(j)$ by its Veronese. We now claim:

Claim 3.5. $A \subseteq Z:=B\left(E^{\prime}, \mathcal{L}, \tau^{\prime}\right)$, for some invertible sheaf $\mathcal{L}$ over an elliptic curve $E^{\prime}$ with $\operatorname{deg} \mathcal{L} \geqslant 2$ and $\left|\tau^{\prime}\right|=\infty$. This embedding may be chosen so that $Z$ is a noetherian left A-module.

Proof of the claim: As was true for $A$, our convention here is that $Z=\oplus_{j \geqslant 0} Z_{n j}$ with

$$
Z_{n j}=H^{0}\left(E^{\prime}, \mathcal{L} \otimes \mathcal{L}^{\tau^{\prime}} \otimes \cdots \otimes \mathcal{L}^{\left(\tau^{\prime}\right)^{j-1}}\right) .
$$


The hypotheses of [AS, Theorem 5.9] are satisfied for $A$, and there is an embedding $A \subseteq Z:=B\left(E^{\prime}, \mathcal{L}, \tau^{\prime}\right) \subset$ $Q_{g r}(A)$ for some ample invertible sheaf $\mathcal{L}$ over some smooth curve $E^{\prime}$ for which $Z$ is a finitely generated left $A$-module. Our choice of grading implies that $Z=Z^{(n)}$ with $\operatorname{dim}_{\mathbb{k}} Z_{n} \geqslant 2$. Thus $\operatorname{deg} \mathcal{L} \geqslant 2$.

By [Sc, Theorem 34], the full division ring of fractions $F:=\operatorname{Fr}(G)$ is finitely generated as both a left and right module over $F^{\prime}:=\operatorname{Fr}(A)$. As in the proof of [RSS1, Proposition 2.6], it follows that $E^{\prime}$ must be elliptic with $\left|\tau^{\prime}\right|=\infty$. Thus the claim is proven.

We return to the proof of the lemma. Since the Goldie rank of the $Q(i)$ as left $A$-modules is bounded above by the finite Goldie rank of $F$ as a left $F^{\prime}$-module, we can remove a finite number of terms and assume that the Goldie rank of the ${ }_{A} Q(i)$ is constant. In particular, each $X(i):=Q(i) Z / Q(0) Z$ is torsion as a left $A$-module. Note that, as ${ }_{A} Z$ is finitely generated, so is each $Q(i) Z$ as a left $A$-module. Similarly, since the $Q(i)$ are finitely generated as right $A$-modules, each $Q(i) Z$ is a finitely generated right $Z$-module. Write $X(i)=\sum_{j=1}^{m} x_{j} Z$ for some $x_{j}$; thus $K(i):=\ell-\operatorname{Ann}_{A}(X(i))=\bigcap_{j} \ell-\operatorname{Ann}_{A}\left(x_{j}\right) \neq 0$. Moreover, AS, Proposition 6.5(2)] implies that $\operatorname{dim}_{\mathbb{k}} A / K(i)<\infty$. In particular, as the $X(i)$ are finitely generated left $A / K(i)$-modules it follows that each $X(i)$ is finite dimensional.

Now consider the $Q(i) Z$ as right $Z$-modules. Since $\operatorname{deg} \mathcal{L} \geqslant 2$, Lemma 2.4 implies that $Z$ is AuslanderGorenstein and CM. By construction, each $Q(i) Z$ is also torsionfree as a right $Z$-module. Thus, by Le, (4.6.6) and Remark 5.8(4)], there is a unique largest essential extension $Y$ of $Q(0) Z$ by finite dimensional right $Z$ modules. By its construction in [Le, $Y$ is finitely generated and hence noetherian as a right $Z$-module. As such, $Y / Q(0) Z$ is finite dimensional, say with right annihilator $L$. Since $Q(0) Z$ is a left $A$-module it follows that, for any $a \in A$, the right $Z$-module $(a Y+Q(0) Z) / Q(0) Z$ is also killed by $L$ and hence is also finite dimensional. Hence $a Y \subseteq Y$ and so $Y=A Y$ is actually a left $A$-module. In particular, as $Y / Q(0) Z$ is finite dimensional, $Y$ is finitely generated as both a right $Z$-module and a left $A$-module.

Finally, as the $X(i)$ are finite dimensional, each $Q(i) Z$ and hence each $Q(i)$ lies in $Y$. Since $A$ is noetherian by [AS, Theorem 0.4], it follows that the union $\bigcup_{n} Q(n)$ is also a noetherian left $A$-module. Thus, the chain $\{Q(n)\}$ must be eventually stationary.

Definition 3.6. For any graded vector subspace $X \subseteq T_{(g)}$, the $g$-divisible hull of $X$ is defined to be

$$
\widehat{X}=\left\{t \in T_{(g)} \mid t g^{n} \in X \text { for some } n \in \mathbb{N}\right\} .
$$

We say that $X$ is $g$-divisible if $X \cap g T_{(g)}=g X$. It is immediate that $\hat{X}$ is $g$-divisible, and if $X$ is $g$-divisible then $\hat{X}=X$. However, even if (say) $X$ is a cg $\mathbb{k}$-algebra, there is no reason for $\hat{X}$ to be cg or even finitely graded.

For any elliptic algebra $T$, the fact that $g T$ is completely prime quickly implies that $T$ is $g$-divisible.

We next prove an important technical result, Lemma 3.9, which should be compared with [RSS2, Proposition 8.7(2)]. The latter proposition gives a similar result in the case when $R \subseteq T$. We begin with a preliminary result. 
Lemma 3.8. Let $E$ be an elliptic curve, with an infinite order automorphism $\sigma$. Let $y \in \mathbb{k}(E)$ and let $V$ be $a \mathbb{k}$-subspace of $\mathbb{k}(E)$ with $2 \leqslant \operatorname{dim} V<\infty$. Then $\operatorname{dim}\left(V+y V^{\sigma}\right)>\operatorname{dim} V$.

Proof. Write $V \cdot \mathcal{O}_{E}$ for the (invertible) subsheaf of the constant sheaf $\mathbb{k}(E)$ generated by $V$. Concretely, $V \cdot \mathcal{O}_{E}=\mathcal{O}\left(D_{V}\right)$, where $D_{V}=\min \{D \in \operatorname{Div}(E): D+(f) \geqslant 0$ for all $f \in V\}$. We have $\left(y V^{\sigma}\right) \cdot \mathcal{O}_{E} \cong \mathcal{O}\left(D_{V}\right)^{\sigma}$. As $\operatorname{deg} D_{V} \geqslant \operatorname{dim} V \geqslant 2$ and $\sigma$ is an infinite-order translation, $\mathcal{O}\left(D_{V}\right)^{\sigma} \nsucceq \mathcal{O}\left(D_{V}\right)$, and thus $y V^{\sigma} \neq V$. The result follows.

Two domains $A$ and $A^{\prime}$ with the same division ring of fractions $\operatorname{Fr}(A)=\operatorname{Fr}\left(A^{\prime}\right)$ are equivalent orders if there exist nonzero elements $a, b, c, d \in \operatorname{Fr}(A)$ such that $a A b \subseteq A^{\prime}$ and $c A^{\prime} d \subseteq A$.

Lemma 3.9. Suppose that $T$ is an elliptic algebra and let $R$ be a noetherian cg algebra with $R \subset\left(T_{(g)}\right)^{(n)}$ for some $n \geqslant 1$. Assume that $g^{n} \in R$ but that $R \nsubseteq \mathbb{k}+g T_{(g)}$. Then the following hold.

(1) Both $\widehat{R}$ and $R^{\prime}:=(\widehat{R})^{(n)}$ are noetherian as left and right $R$-modules, with $g^{m n} R^{\prime} \subseteq R \subseteq R^{\prime}$ for some $m$.

(2) In particular, $\widehat{R}$ and $R^{\prime}$ are noetherian $c g \mathbb{k}$-algebras while $R^{\prime}$ is a equivalent order to $R$.

Proof. Note that Part (2) is an immediate consequence of Part (1) combined with the observation from Remark 2.2(2) and so only Part (1) needs proof.

In this result, we regard $\left(T_{(g)}\right)^{(n)}$ as a subalgebra of $T_{(g)}$ and do not change the grading. We start by simplifying the problem. Since $R \nsubseteq \mathbb{k}+g T_{(g)}$, there exists some $x \in R_{a n} \backslash g T$. For the moment consider $U:=R^{(a n)}$, which is also noetherian by [AS, Lemma 4.10(2)]. Suppose that $\hat{U}$ is noetherian as both a left and a right $U$-module. Note that if $r \in R_{n s}$ then $r g^{n \ell} \in U$ for the appropriate $\ell$ and so $R \subseteq \widehat{U}$ and hence $\widehat{R}=\widehat{U}$. Thus $\hat{R}$ is a noetherian module over both $U$ and $R$. Therefore, we may replace $R$ by $U$ and $n$ by an and assume that there exists $x \in R_{n} \backslash g T$.

Next, set $C:=R[g]$. Since $g^{n} \in R$, this is certainly a noetherian $R$-module with $\widehat{C}=\widehat{R}$. In other words, $R^{\prime}=\widehat{C}^{(n)}$. Moreover, since $R \subset\left(T_{(g)}\right)^{(n)}$, clearly $C^{(n)}=R\left[g^{n}\right]=R$. Note, also, that

$$
\widehat{C}^{(n)}=\left\{x \in\left(T_{(g)}\right)^{(n)}: g^{n \ell} x \in R \text { for some } \ell \geqslant 1\right\} .
$$

For $x \in T_{(g)}$, let $\bar{x}=x+g T_{(g)} \in T_{(g)} / g T_{(g)}$. Set $B=\bar{R}=\left(R+g T_{(g)}\right) / g T_{(g)}$. For $i \geqslant 0$, define

$$
Q(i):=\frac{\left(g^{-i n} C \cap T_{(g)}\right)+g T_{(g)}}{g T_{(g)}} \subseteq \frac{T_{(g)}}{T_{(g)} g} .
$$

As $g^{n} \in R$, clearly $\bar{C}=Q(0)$ with $Q(j) \subseteq Q(j+1) \subseteq \bigcup_{i \geqslant 0} Q(i)=\overline{\widehat{C}}$ for $j \geqslant 0$.

The next sublemma provides the strategy for the proof of the lemma.

Sublemma 3.11. In the above notation, suppose that $Q(r)=Q(r+1)$ for $r \geqslant r_{0}$. Then $\widehat{R}=\hat{C}$ is an equivalent order to $C$ and is noetherian as a one-sided $C$-module and hence as a one-sided $R$-module.

Moreover, $R^{\prime}=\widehat{C}^{(n)}$ is an equivalent order to $R$, with $g^{\ell n} R^{\prime} \subseteq R \subseteq R^{\prime}$ for some $\ell \geqslant 1$. As such, $R^{\prime}$ is a noetherian cg $\mathbb{k}$-algebra. 
Proof of the Sublemma. The proof is essentially the same as the second paragraph of the proof of RSS2, Proposition 8.7(1)], although for the reader's convenience we include a proof here.

To begin with, we claim that $\widehat{C} \cap g^{m} T_{(g)}=C \cap g^{m} T_{(g)}$ for all $m \geqslant \rho=r_{0} n$. If not, there exists $y:=g^{m} x \in\left(\widehat{C} \cap g^{m} T_{(g)}\right) \backslash C$ for some such $m$. Choose $(y, x)$ with this property for which $x$ has minimal degree. This ensures that $y \notin g^{m+1} T_{(g)}$, since otherwise one could write $y=g^{m+1} x^{\prime}$ with $\operatorname{deg}\left(x^{\prime}\right)=\operatorname{deg}(x)-1$. Note that, as $g^{m} x \in \widehat{C}$, certainly $g^{m+\ell} x \in C$ for some $\ell$, and so $x \in \widehat{C}$. Therefore, we can write $\bar{x}=\left[x+g T_{(g)}\right]$ as $\bar{x}=\bar{w}$ for some $w \in g^{-\rho} C \cap T_{(g)}$. Thus $g^{m} w=g^{m-\rho}\left(g^{\rho} w\right) \in C$. Moreover, $x-w=g t$ for some $t \in T_{(g)}$ and so $g^{m+1} t=g^{m} x-g^{m} w \in g^{m} T_{(g)} \cap \widehat{C}$. Here, $\operatorname{deg} t=\operatorname{deg} x-1$ and so, by the inductive hypothesis for $(y, x)$, we obtain $g^{m+1} t \in C$. In other words, $g^{m} x=g^{m} w+g^{m+1} t \in C$; a contradiction. Thus the claim is proven.

By the claim, $I:=\widehat{C} \cap g^{\rho} T_{(g)}$ is a non-zero ideal of both $C$ and $\widehat{C}$, and so certainly $C$ and $\widehat{C}$ are equivalent orders. As $g \widehat{C}=\widehat{C} \cap g T_{(g)}$, an easy induction shows that $g^{\rho} \widehat{C}=\widehat{C} \cap g^{\rho} T_{(g)}=C \cap g^{\rho} T_{(g)}$. Thus, $g^{\rho} \widehat{C} \subseteq I \subset C$ and so $\widehat{R}=\widehat{C}$ is a noetherian $C$-module and hence a noetherian $R$-module on both sides, while it is cg by Remark 2.2. Moreover, $g^{\rho} R^{\prime}=g^{\rho} \widehat{C}^{(n)} \subseteq C^{(n)}=R \subseteq R^{\prime}$ and so $R^{\prime}$ and $R$ are indeed equivalent orders.

Returning to the proof of the lemma note that, by the second paragraph of the proof, there exists $0 \neq x \in B_{n}$. Suppose first that $\operatorname{dim}_{\mathbb{k}} Q(i)_{j} \leqslant 1$ for all $i, j$. As $B$ and the $Q(i)$ are contained in the domain $T_{(g)} / g T_{(g)} \cong \mathbb{k}(E)\left[z, z^{-1} ; \tau\right]$, this implies that $B$ contains the domain $\mathbb{k}[x]$. Moreover, $W:=\bigcup Q(i)$ satisfies $\operatorname{dim}_{\mathbb{k}} W_{n} \leqslant 1$ for all $n \geqslant 0$. As such, $W$ is finitely generated as a $k[z]$-module and hence as a $B$-module. In particular, $Q(r)=Q(r+1)$ for all $r \gg 0$ and so the lemma follows from Sublemma 3.11. (In fact a little more work shows that this case cannot happen.)

We may therefore assume that there exists $i, j \geqslant 1$ such that $V:=Q(i)_{j}$ has $\operatorname{dim} V \geqslant 2$. We next show that this implies that GKdim $B=\operatorname{GKdim} \bar{C}=2$. Since $C^{(n)}=R$, clearly $(\bar{C})^{(n)}=\bar{R}^{(n)}=B$, and so it suffices to prove that $\mathrm{GK} \operatorname{dim} \bar{C}=2$. Note that, as $C$ is a noetherian $R$-module, each $Q(i)$ is finitely generated as a $C$-module and hence as both an $R$-module and a $B$-module on either side. Now $Q(i)_{k+n} \supseteq x Q(i)_{k}+Q(i)_{k} x$ for all $k \in \mathbb{Z}$. Since each $Q(i) \subseteq \mathbb{k}(E)\left[z, z^{-1} ; \tau^{n}\right]$, we may apply Lemma 3.8 with $\sigma=\tau^{n}$, to show that $\operatorname{dim} Q(i)_{j+n a} \geqslant \operatorname{dim} V+a$ for all $a \geqslant 1$. Hence $\operatorname{GKdim} Q(i) \geqslant 2$. As $Q(i)$ is a finitely generated left and right $\bar{C}$-module it follows that $\operatorname{GK} \operatorname{dim} B=\operatorname{GK} \operatorname{dim} \bar{C} \geqslant 2$.

Conversely, we know that $\bar{C}$ is a noetherian, cg subalgebra of $T_{(g)} / g T_{(g)} \cong \mathbb{k}(E)\left[z, z^{-1} ; \tau\right]$, and hence of $\mathbb{k}(E)[z ; \tau]$. It is therefore a finitely generated algebra by the graded Nakayama's Lemma. Thus, by [AS, Theorem 0.1], GKdim $\bar{C} \leqslant 2$. Combined with the last paragraph, this implies that GKdim $B=$ $\operatorname{GKdim} \bar{C}=2$.

We can now apply Lemma 3.4 to $A=B$ with $B \subseteq Q(i) \subseteq Q(i+1) \subseteq T_{(g)} / g T_{(g)} \cong k(E)\left[z, z^{-1} ; \tau\right]$. Thus, by that result, $Q(r)=Q(r+1)$ for all $r \gg 0$ and so we can apply the sublemma. Since $R \subseteq R^{\prime}$, the lemma follows. 


\section{OverRings of LOCALLY SIMPLE ELLIPTIC ALGEBRAS}

In this section we begin to work towards Theorem 1.5. We fix an elliptic algebra $T \subset T_{(g)}$ with factor $B=T / g T$ and localisation $T^{\circ}=T\left[g^{-1}\right]_{0}$ and then isolate conditions that preclude the existence of proper noetherian cg overrings of $T$. These involve slightly awkward conditions (see Hypothesis 4.1) that will be refined in later sections.

We first comment on the title to this section: we define $T$, or any other elliptic algebra, to be locally simple if the localisation $T^{\circ}$ is a simple ring. The intuition behind this term is that if one regards qgr- $T$ as the category of coherent sheaves on the noncommutative (and non-existent) projective variety Proj $T$ then $\bmod -T^{\circ}$ corresponds to the analogous category of sheaves on the noncommutative affine space Proj $T \backslash E$. This is, in turn, the smallest nonempty open subset of $\operatorname{Proj} T$.

Hypothesis 4.1. Assume that:

(1) The ring $T^{\circ}$ is a simple domain with division ring of fractions $\operatorname{Fr}\left(T^{\circ}\right)$.

(2) There is a fixed $\mathbb{Z}$-graded overring $T \varsubsetneqq R \subset T_{(g)}$ and a fixed simple right $T^{\circ}$-module $\mathcal{S} \subseteq R^{\circ} / T^{\circ}$.

(3) There exists an extension $0 \rightarrow \mathcal{S} \rightarrow X(\mathcal{S}) \rightarrow y \rightarrow 0$ of right $T^{\circ}$-modules of finite length such that:

(a) $X:=X(\mathcal{S})$ has finite projective dimension;

(b) $\mathcal{S}=\operatorname{Soc}(X)$.

(4) $\mathcal{S}$ cannot be written as the localisation $\mathcal{S}=L^{\circ}$ of a $T$-line module $L$.

We make a few comments about these hypotheses. First, we do not assume here that $X(\mathcal{S})$ is unique. However, if $\operatorname{pd}(\mathcal{S})<\infty$, in which case $(3)$ holds automatically, we always set $\mathcal{X}(\mathcal{S})=\mathcal{S}$. Finally, note that the hypotheses imply that $\mathcal{S}$ is essential in $X$.

We first note some elementary properties of modules over rings of injective dimension one.

Lemma 4.2. Let $A$ be a noetherian (or Goldie) prime ring of injective dimension one. Then

(1) Every torsionfree, finitely generated right A-module $P$ of finite projective dimension is projective.

(2) Every finitely generated torsionfree and every finitely generated torsion A-module is CM.

Proof. (1) It does no harm to replace $P$ by some direct sum $P^{r}$ so that $P$ has Goldie rank equal to an integer $t$ times the Goldie rank of $A$. If $F=\operatorname{Fr}(A)$ we can then identify $P \subseteq P F=F^{t}$. Clearing denominators on the left gives an embedding of $P$ into the finitely generated, free right $A$-module $A^{t}$. Set $M=A^{t} / P$, which is therefore a torsion module. Then $n:=\operatorname{pd}(M)<\infty$, say with $\operatorname{Ext}_{A}^{n}(M, N) \neq 0$ for some finitely generated module $N$. If $A^{m} \rightarrow N$ then, from the usual long exact sequence in cohomology, $\operatorname{Ext}_{A}^{n}\left(M, A^{m}\right) \neq 0$, as well. Thus $n \leqslant 1$ by hypothesis and so $P$ is projective.

(2) If $M$ is a finitely generated torsion right $A$-module then $\operatorname{Ext}_{A}^{n}(M, A) \neq 0$ if and only if $n=1$. If $P$ is a torsionfree right $A$-module, then it is again harmless to replace $P$ by some $P^{r}$. Then, as in the proof of (1) 
we can embed $P$ into a free right $A$-module $A^{t}$ so that $M=A^{t} / P$ is torsion. It follows that $\operatorname{Ext}_{A}^{n}(P, A) \neq 0$ if and only if $n=0$.

We next apply these properties to localisations of elliptic algebras.

Lemma 4.3. Let $T$ be an elliptic algebra. Then $T^{\circ}$ is Auslander-Gorenstein and Cohen-Macaulay with $\operatorname{inj\operatorname {dim}}\left(T^{\circ}\right) \leqslant 2$. If in addition $T^{\circ}$ is simple, then

(1) $T^{\circ}$ has injective dimension $\operatorname{inj} \operatorname{dim}\left(T^{\circ}\right)=1$.

(2) Every torsionfree, finitely generated right $T^{\circ}$-module $\mathcal{P}$ of finite projective dimension is projective.

(3) Every finitely generated torsionfree and every finitely generated torsion $T^{\circ}$-module is $C M$.

(4) In particular, if $T^{\circ}$ satisfies the conditions of Hypothesis 4.1, then $\operatorname{pd}(\mathcal{X})=1$.

Proof. By [RSS1, Lemmas 2.1 and 2.2], $T^{\circ} \cong T /(g-1) T$, which has a natural filtration $\Lambda$ induced from the graded structure of $T$. As such, the associated graded ring $\operatorname{gr}_{\Lambda} T \cong T / g T=B$. By [Le, Theorem 6.6], $B$ is Auslander-Gorenstein and CM of injective dimension 2. Thus, by [Bj, Theorem 4.1 and its proof], $T^{\circ}$ is also Auslander-Gorenstein and $\mathrm{CM}$ with $\operatorname{inj\operatorname {dim}}\left(T^{\circ}\right) \leqslant 2$.

Now for (1), if $T^{\circ}$ is simple, it has no finite dimensional modules, so the CM condition easily implies that $\operatorname{Ext}_{T^{\circ}}^{2}\left(M, T^{\circ}\right)=0$ for all finitely generated right $T^{\circ}$-modules $M$, and hence that injdim $\left(T^{\circ}\right)<2$, as required. Parts (2) and (3) follow from (1) combined with Lemma 4.2, while (4) is immediate from (2).

As a partial converse to Lemma 4.3 we have the following well-known result, for which we could not find an appropriate reference.

Lemma 4.4. Let $A$ be a finitely generated $\mathbb{k}$-algebra that is a prime noetherian, Auslander-Gorenstein and $C M$ ring, with $\operatorname{GKdim}(A)=2$. If $A$ is also hereditary, then $A$ is simple.

Proof. If $A$ is not simple, pick a prime ideal $P \neq 0$ of $A$. Then $\operatorname{GKdim}(A / P) \leqslant 1$ by [KL, Proposition 3.5]. Thus, $A / P$ satisfies a polynomial identity by [SW]. As such, $A / P$ and hence $A$ has a finite dimensional factor $\operatorname{ring} \bar{A}\left[\mathrm{KL}\right.$, Corollary 10.9]. But now the $\mathrm{CM}$ condition implies that $j_{A}(\bar{A})=2$, whence $\operatorname{Ext}_{A}^{2}(\bar{A}, A) \neq 0$. This contradicts the hereditary assumption.

We next give a general lemma on torsionfree extensions, which we will use several times below.

Lemma 4.5. Let $A$ be a prime right noetherian ring and let $L$ be an essential submodule of the finitely generated right $A$-module $M$. Further suppose that $\operatorname{Ext}_{A}^{2}(M / L, A)=0$. Then every torsionfree extension $0 \rightarrow A \rightarrow X \rightarrow L \rightarrow 0$ lifts to an extension $0 \rightarrow A \rightarrow Y \rightarrow M \rightarrow 0$ and every such $Y$ is torsionfree.

Proof. Let $\alpha: \operatorname{Ext}_{A}^{1}(M, A) \rightarrow \operatorname{Ext}_{A}^{1}(L, A)$ be the map induced from the inclusion $L \subseteq M$. As $\alpha$ is surjective by the assumption on $M / L$, if we regard $X$ as an element of $\operatorname{Ext}_{A}^{1}(L, A)$, then there exists $Y \in \alpha^{-1}(X)$. We may assume that $A \subseteq X \subseteq Y$. Let $Z$ be the torsion submodule of $Y$, and suppose $Z \neq 0$. Because $X$ is torsionfree, $Z \cap X=0$. Thus $(Z+A) \cap X=(Z \cap X)+A=A$. Now $Y / A \supseteq(Z+A) / A \neq 0$ and $X / A \cong L$ 
is essential in $Y / A \cong M$, from which it follows that $((Z+A) / A) \cap X / A \neq 0$. Thus $(Z+A) \cap X \supsetneqq A$, a contradiction.

Lemma 4.6. Assume that $T \varsubsetneqq R$ satisfy Hypothesis 4.1. Let $R^{\circ} \supseteq \mathcal{C} \supset T^{\circ}$ be a (torsionfree) $T^{\circ}$-module such that $\mathcal{C} / T^{\circ} \cong \mathcal{S}$. Then there exists a projective $T^{\circ}$-module $\breve{\mathcal{C}}$ such that $\operatorname{Fr}\left(T^{\circ}\right) \supset \check{\mathcal{C}} \supseteq \mathcal{C}$ and $\breve{\mathcal{C}} / T^{\circ} \cong X(\mathcal{S})=X$.

Proof. If $\mathcal{C}$ is projective then $\operatorname{pd}(\mathcal{S})<\infty$ and $\mathcal{X}=\mathcal{S}$. Thus $\check{\mathcal{C}}=\mathcal{C}$, which is automatically projective, as required. So assume that $\mathcal{C}$ is not projective.

Take the short exact sequence $0 \longrightarrow \mathcal{S} \stackrel{a}{\longrightarrow} x \stackrel{b}{\longrightarrow} y \longrightarrow 0$ given by Hypothesis 4.1 noting that $\mathcal{S}$ is essential in $X$. By Lemma $4.3, A=T^{\circ}$ has injective dimension 1 and so, by Lemma 4.5, $\mathcal{C}$ lifts to an extension $0 \rightarrow T^{\circ} \rightarrow \mathcal{M} \rightarrow \mathcal{X} \rightarrow 0$, in which $\mathcal{M}$ is torsionfree. This also implies that there is a natural

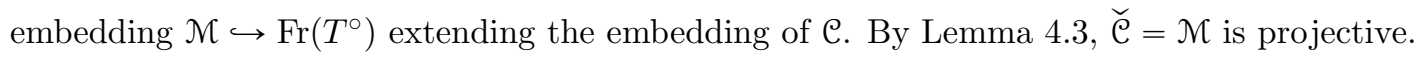

Notation 4.7. Let $\mathcal{M}$ be a $T^{\circ}$-submodule of $\operatorname{Fr}\left(T^{\circ}\right)$. Following [RSS2, Section 7, p.2099] we define $\Phi \mathcal{M}:=$ $\oplus_{n \in \mathbb{Z}}(\Phi \mathcal{M})_{n}$, where $(\Phi \mathcal{N})_{n}:=\left\{a \in\left(T_{(g)}\right)_{n}: a g^{-n} \in \mathcal{M}\right\}$. As $T$ is $g$-divisible, it is immediate that $T=\Phi\left(T^{\circ}\right)$ and hence that $\Phi \mathcal{M}$ is a $T$-module. We remark that [RSS2] used $\Omega$ in place of $\Phi$, but in this paper $\Omega$ will be reserved for another more longstanding construction.

Let $X$ be a $T$-submodule of $T_{(g)}$ and recall the definition of the $g$-divisible hull $\hat{X}$ from Definition 3.6 We note that $\hat{X}=\Phi\left(X^{\circ}\right)$.

We are now ready to prove the first main result on the non-existence of finitely graded overrings; indeed the main theorems from the introduction will ultimately reduce to this case. We note that the idea of the proof originates in Goodearl's result [Go, Theorem 5] that overrings of HNP rings are localisations.

Proposition 4.8. Assume that $T \varsubsetneqq R$ satisfies the conditions of Hypothesis 4.1.

(1) If $R$ is $g$-divisible, then $\operatorname{dim} R_{0}=\infty$.

(2) If $R$ is finitely graded, then $R$ is not noetherian.

Proof. (1) It is immediate that $\Phi\left(R^{\circ}\right)=\widehat{R}=R$. Now let $T^{\circ} \subset \mathcal{C} \subset R^{\circ}$ be a $T^{\circ}$-module with $\mathcal{C} / T^{\circ} \cong \mathcal{S}$. By Hypothesis 4.1(4), Proposition [3.2(2) applies and provides a $T$-module extension $T \varsubsetneqq D \subset T_{(g)}$ with $D^{\circ} / T^{\circ} \cong \mathcal{S}$, and so that $D_{r} \supsetneqq T_{r}$ for some $r \leqslant 0$. Let $\mathcal{D}:=D^{\circ}$.

Apply Lemma 4.6. This provides extensions

$$
T^{\circ} \varsubsetneqq \mathcal{C} \subset \breve{\mathrm{C}} \subset \operatorname{Fr}\left(T^{\circ}\right) \quad \text { and } \quad T^{\circ} \subset \mathcal{D} \subset \breve{\mathcal{D}} \subset \operatorname{Fr}\left(T^{\circ}\right)
$$

such that $\mathcal{C} / T^{\circ}=\operatorname{Soc}\left(\breve{\mathcal{C}} / T^{\circ}\right)$ and $\mathcal{D} / T^{\circ}=\operatorname{Soc}\left(\breve{\mathcal{D}} / T^{\circ}\right)$ and there is an isomorphism

$$
\chi: \check{\mathcal{C}} / T^{\circ} \stackrel{\cong}{\Longrightarrow} x \stackrel{\cong}{\longrightarrow} / T^{\circ} .
$$

(If $\mathcal{S}$ and hence $\mathcal{C}$ have finite projective dimension then $X=\mathcal{S}$ whence $\breve{\mathcal{C}}=\mathcal{C}$ and $\breve{\mathcal{D}}=\mathcal{D}$. In this case the desired properties hold tautologically.) 
Let $\pi_{1}: \check{\mathcal{C}} \rightarrow \breve{\mathcal{C}} / T^{\circ}$ and $\pi_{2}: \check{\mathcal{D}} \rightarrow \breve{\mathcal{D}} / T^{\circ}$ be the natural projections. By Lemma 4.6, $\breve{\mathcal{C}}$ is projective, and so the isomorphism $\chi$ lifts to a $T^{\circ}$-module homomorphism $\xi: \breve{\mathcal{C}} \rightarrow \breve{\mathcal{D}}$ making the following diagram commute.

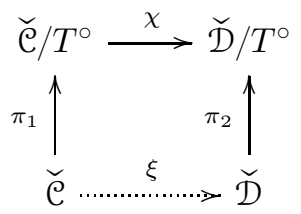

As $\breve{\mathcal{C}}$ and $\breve{\mathcal{D}}$ are submodules of $\operatorname{Fr}\left(T^{\circ}\right), \xi$ is given by left multiplication by an element $x \in \operatorname{Fr}\left(T^{\circ}\right)$. Since $\chi \pi_{1}\left(T^{\circ}\right)=0$ the commutativity of (4.9) implies that $\pi_{2} \xi\left(T^{\circ}\right)=0$ and $\xi\left(T^{\circ}\right) \subseteq T^{\circ}$. In other words, $x \in T^{\circ}$.

Next, since $\mathcal{C} / T^{\circ}=\operatorname{Soc}\left(\breve{\mathcal{C}} / T^{\circ}\right)$, and $\pi_{2} \xi=\chi \pi_{1}$ is surjective, the map $\xi$ must map $\mathcal{C}$ to the preimage of the simple socle $\operatorname{Soc}\left(\breve{\mathcal{D}} / T^{\circ}\right)=\mathcal{D} / T^{\circ}$. Consequently, $\mathcal{D} / T^{\circ}=\left(x \mathcal{C}+T^{\circ}\right) / T^{\circ}$. In particular, since $R^{\circ}$ is a ring, $\mathcal{D}=\left(x \mathcal{C}+T^{\circ}\right) \subseteq x R^{\circ}+T^{\circ} \subseteq R^{\circ}$. Since $\mathcal{D}=D^{\circ}$, it follows that $D \subseteq \Phi\left(R^{\circ}\right)=R$, and so, by Remark 2.2 $(1)$, $\operatorname{dim} R_{0}=\infty$.

(2) Suppose that $R$ is finitely graded and noetherian. Then, by Lemma 3.9 $\widehat{R}$ is a finitely generated right (and left) $R$-module and so $\widehat{R}$ is also finitely graded. Moreover, by construction, $\widehat{R}^{\circ}=R^{\circ}$ and so Hypothesis 4.1 holds for $\widehat{R}$. Thus we can apply Part (1) to $\widehat{R}$ to show that $\widehat{R}_{0}$ is infinite dimensional; a contradiction.

We remark that in the penultimate sentence of the proof of Part (1) of Proposition 4.8 one needs only that $R^{\circ}$ is a $T^{\circ}$-bimodule. In other words, the argument above proves the following statement. Since the result will not be used in the paper, the details are left to the interested reader.

Corollary 4.10. Assume that $T \varsubsetneqq R$ satisfies the conditions of Hypothesis 4.1 . Let $\mathcal{E} \subseteq \operatorname{Fr}\left(T^{\circ}\right)$ be the maximal extension of $T^{\circ}$ by a direct sum of copies of $\mathcal{S}$, and note that $\mathcal{E}$ is a $T^{\circ}$-bimodule. Then $\mathcal{E} / T^{\circ}$ is simple as a $T^{\circ}$-bimodule.

\section{Algebras with $A_{1}$ singularities}

In the next section we will show that Proposition 4.8 can be applied to show that the quadric algebras $Q$ defined by Van den Bergh in VB3] are indeed minimal elliptic surfaces. The corresponding algebra $Q^{\circ}$ will either have finite global dimension or be simple with a mild singularity. In this section we prepare for the latter case by studying arbitrary elliptic algebras $T$ for which $T^{\circ}$ has such a singularity. The relevant definitions are as follows. As usual, given a right (left) module $M$ over a ring $A$ we write $M^{*}$ for the left (right) $A$-module $\operatorname{Hom}_{A}(M, A)$.

Definition 5.1. Given a noetherian ring $A$ of finite injective dimension define the singularity category $\operatorname{Sing}(A)$ as in $[\mathrm{AB}$, Section I.5, p.46] (where it is called the Stabilised Category) and write Hom groups in $\operatorname{Sing}(A)$ by $\underline{\operatorname{Hom}}_{A}(M, N)$, for right $A$-modules $M$ and $N$. By [Bu, Theorem 4.4.1], $\operatorname{Sing}(A)$ can be identified with the quotient category

$$
\operatorname{Sing}(A) \simeq\{\text { maximal Cohen-Macaulay }(\mathrm{MCM}) \text { modules }\} / \text { projective modules }\}
$$


For right MCM modules $M, N$ we have, by [Bu, 2.1],

$\underline{\operatorname{Hom}}_{A}(M, N)=\operatorname{Hom}_{A}(M, N) /\{$ maps factoring through a projective module $\}=\operatorname{Hom}_{A}(M, N) / N M^{*}$, where $N M^{*}$ is the natural image of $N \otimes M^{*}$ inside $\operatorname{Hom}_{A}(M, N)$. By [Bu, Theorem 4.4.1], $\operatorname{Sing}(A)$ is triangulated with the inverse of the homological shift $\Sigma$ given by the syzygy functor $\Omega$.

We say that $A$ has an $A_{1}$ singularity if $\operatorname{Sing}(A)$ is triangle equivalent to the category of $\mathbb{k}$-vector spaces Vect (necessarily with trivial homological shift). If char $\mathbb{k} \neq 2$, then the Kleinian singularity $\mathbb{k}[[x, y]]^{C_{2}}$ has an $A_{1}$ singularity, whence the name.

In the next few results, we give some basic properties of rings with $A_{1}$ singularities.

Remark 5.2. Observe that if an algebra $A$ has an $A_{1}$ singularity, then there exists an MCM right $A$-module $\mathcal{M}$ (equivalently, by Lemma 4.2, a finitely generated torsionfree module $\mathcal{M}$ ), so that

(a) $\mathcal{M}$ corresponds to $\mathbb{k}$ under the equivalence $\operatorname{Sing}(A) \simeq$ Vect,

(b) $\underline{\operatorname{End}}_{A}(\mathcal{M})=\operatorname{End}_{A}(\mathcal{M}) / \mathcal{M} \mathcal{M} \mathcal{M}^{*} \cong \mathbb{k}$, and

(c) the first syzygy $\Omega \mathcal{N} \cong \mathcal{M}$ in $\operatorname{Sing}(A)$.

We refer to $\mathcal{M}$ as the generator of $\operatorname{Sing}(A)$.

Lemma 5.3. Let $A$ be a prime noetherian ring of injective dimension 1 that is either hereditary or has an $A_{1}$ singularity. Let $\mathcal{N}$ be a non-zero, finitely generated, torsionfree right $A$-module. Let $\mathcal{N}$ generate $\operatorname{Sing}(A)$ if $\operatorname{Sing}(A)$ is nontrivial, or else let $\mathcal{M}=A$. Then the following hold.

(1) There exist an integer $s \geqslant 0$ and finitely generated projective right $A$-modules $\mathcal{A}$ and $\mathcal{B}$ such that $\mathcal{N} \oplus \mathcal{A} \cong \mathcal{B} \oplus \mathcal{M}^{(s)}$.

(2) There exist a projective right $A$-module $\mathcal{L}$ and a short exact sequence $0 \rightarrow \mathcal{N} \rightarrow \mathcal{L} \rightarrow \mathcal{N} \rightarrow 0$. In particular, this is true for $\mathcal{N}=\mathcal{M}$.

Proof. We may assume that $\operatorname{Sing}(A)$ is nontrivial.

(1) By Remark [5.2(a) there exists $s \geqslant 0$ such that $\mathcal{N} \cong \mathcal{M}^{(s)}$ in $\operatorname{Sing}(A)$. Now apply AB , Proposition 1.44(4)].

(2) The result is trivial if $\mathcal{N}$ is projective, so assume not. Pick an isomorphism $\mathcal{N} \cong \mathcal{M}^{(s)}$ in $\operatorname{Sing}(A)$ by Part (1) and note that $s \geqslant 1$ as $\mathcal{N}$ is not projective. Choose a surjection $\chi: \mathcal{P} \rightarrow \mathcal{N}$ where $\mathcal{P}$ is a finitely generated projective module and set $\mathcal{N}^{\prime}=\operatorname{Ker}(\chi)$. By Remark $5.2(\mathrm{c}), \mathcal{N}^{\prime} \cong(\Omega \mathcal{N})^{(s)} \cong \mathcal{M}^{(s)} \cong \mathcal{N}$ in $\operatorname{Sing}(A)$. Thus there are projective modules $\mathcal{A}$ and $\mathcal{B}$ so that $\mathcal{N}^{\prime} \oplus \mathcal{A} \cong \mathcal{N} \oplus \mathcal{B}$.

From the short exact sequence $0 \rightarrow \mathcal{N}^{\prime} \rightarrow \mathcal{P} \rightarrow \mathcal{N} \rightarrow 0$ we obtain a short exact sequence

$$
0 \longrightarrow \mathcal{N}^{\prime} \oplus \mathcal{A} \longrightarrow \mathcal{P} \oplus \mathcal{A} \longrightarrow \mathcal{N} \longrightarrow 0
$$

By the previous paragraph, this induces an exact sequence

$$
0 \longrightarrow \mathcal{N} \oplus \mathcal{B} \stackrel{\alpha}{\longrightarrow} \mathcal{P} \oplus \mathcal{A} \stackrel{\beta}{\longrightarrow} \mathcal{N} \rightarrow 0 .
$$


Set $\mathcal{L}:=(\mathcal{P} \oplus \mathcal{A}) / \alpha(\mathcal{B})$. Then

$$
\mathcal{L} \supseteq \mathcal{Z}:=\frac{\alpha(\mathcal{N}) \oplus \alpha(\mathcal{B})}{\alpha(B)} \cong \mathcal{N} \quad \text { with } \quad \mathcal{L} / \mathcal{Z} \cong \frac{\mathcal{P} \oplus \mathcal{A}}{\alpha(\mathcal{N} \oplus \mathcal{B})} \cong \mathcal{N} .
$$

Finally, by its initial construction $\operatorname{pd}(\mathcal{L}) \leqslant 1$ and from the last displayed equation it follows that $\mathcal{L}$ is torsionfree. Thus, by Lemma $4.2, \mathcal{L}$ is projective, as required.

Lemma 5.4. Let $A$ be a prime noetherian ring of injective dimension 1 that is either hereditary or has an $A_{1}$ singularity. Let $\mathcal{C}$ be a right $A$-module with $A \subset \mathcal{C} \subset \operatorname{Fr}(A)$ and such that $\mathcal{C} / A \cong \mathcal{S}$ is a simple right A-module.

Then either $\operatorname{pd}(\mathcal{S})<\infty$ or there exists a finitely generated right $A$-module $\mathcal{X}=X(\mathcal{S})$ of finite projective dimension for which there exists an extension

$$
0 \longrightarrow \mathcal{S} \longrightarrow X \longrightarrow \mathcal{S} \longrightarrow 0
$$

Proof. We may assume that $\operatorname{pd}(\mathcal{S})=\infty$. By Lemma $5.3(2)$ we have a short exact sequence

$$
0 \longrightarrow \mathcal{C}_{1} \stackrel{\alpha}{\longrightarrow} \mathcal{L} \stackrel{\beta}{\longrightarrow} \mathcal{C}_{2} \longrightarrow 0
$$

with $\mathcal{L}$ projective and the $\mathcal{C}_{i}$ being the two copies of $\mathcal{C}$. The natural inclusion of $A$ into $\mathcal{C}_{2}$ lifts to a monomorphism $\theta: A \rightarrow \mathcal{L}$. Thus if $\iota(A)$ is the given copy of $A$ inside $\mathcal{C}_{1}$ then $\iota(A) \oplus \theta(A) \subset \mathcal{L}$. Clearly,

$$
\frac{\mathcal{L}}{(\iota(A) \oplus \theta(A))+\mathcal{C}_{1}} \cong \frac{\mathcal{C}_{2}}{A}=\mathcal{S}
$$

while

$$
z:=\frac{(\iota(A) \oplus \theta(A))+\mathcal{C}_{1}}{\iota(A) \oplus \theta(A)}
$$

is a homomorphic image of $\mathcal{C}_{1} / \iota(A) \cong \mathcal{S}$. Since $\mathcal{S}$ is simple and $\mathcal{Z} \neq 0$, this implies that $\mathcal{Z} \cong \mathcal{S}$.

Finally, consider $X:=\mathcal{L} /(\iota(A) \oplus \theta(A))$. Then, clearly $\operatorname{pd}(X) \leqslant 1$, while the last paragraph ensures that there is the required short exact sequence $0 \rightarrow \mathcal{S} \rightarrow X \rightarrow \mathcal{S} \rightarrow 0$.

Applying the results of this section to elliptic algebras we obtain our first result on minimal elliptic surfaces:

Theorem 5.5. Let $T$ be an elliptic algebra with localisation $T^{\circ}$. Assume either that $T^{\circ}$ is hereditary or that $T^{\circ}$ is simple with an $A_{1}$ singularity. Further assume that $T$ has no line modules. Then $T$ is a minimal elliptic surface.

More generally, let $T \subseteq R \subset T_{(g)}$ be a graded overring. Then:

(1) if either $\widehat{R}$ is finitely graded or $R$ is both finitely graded and noetherian, then $R=T$;

(2) if $T \neq R$ and $R=\widehat{R}$ is g-divisible, then $\operatorname{dim} R_{0}=\infty$.

Remark 5.6. If $T^{\circ}$ is hereditary and $\operatorname{deg} T \geqslant 3$, then the conclusion of theorem still holds provided $T$ has no line modules of self-intersection $(-1)$. See Theorem 8.12 for the details. 
Proof. Note that $T^{\circ}$ is a finitely generated $\mathbb{k}$-algebra by [RSS1, Lemma 2.1]. Thus, if $T^{\circ}$ is hereditary, then $T^{\circ}$ is also simple by Lemma 4.4 .

Pick a simple $T^{\circ}$-submodule $\mathcal{S} \subseteq R^{\circ} / T^{\circ}$. If $\mathcal{S}$ has infinite projective dimension then, by Lemma 5.4, the conditions of Hypothesis 4.1 are satisfied. If $\operatorname{pd}(\mathcal{S})<\infty$, then Hypothesis 4.1 is immediate. In either case, the result follows from Proposition 4.8 ,

We note the following curious consequence of the theorem, which seems to us a strikingly weird result:

Corollary 5.7. Let $T$ be as in Theorem 5.5 and let $R$ be the subalgebra of $T_{(g)}$ generated by $T$ and $x$, for any homogeneous element $x \in T_{(g)} \backslash g T_{(g)}$ of positive degree. Then:

(1) $R$ is not noetherian;

(2) $\widehat{R}_{0}$ is infinite dimensional.

Finally, we note for future reference the following sufficient condition, due to Simon Crawford, for when a ring has an $A_{1}$ singularity. Note that the hypothesis that $M$ is a generator is automatic if $A$ is simple.

Proposition 5.8. ( $[\mathrm{Cr}$, Theorem 4.5.7]) Let $A$ be a left and right noetherian $\mathbb{k}$-algebra of injective dimension at most 2. Suppose that $A$ has an $M C M$ right module $M$ which is a generator such that $\operatorname{gldim} \operatorname{End}_{A}(M) \leqslant 2$ and $\underline{\operatorname{End}}_{A}(M)=\operatorname{End}_{A}(M) / M M^{*} \cong \mathbb{k}$. Then $\operatorname{gldim} A=\infty$ and $A$ has an $A_{1}$ singularity.

\section{Sklyanin algebras and Van den Bergh quadrics}

In this section we apply Theorem 5.5 to some well-known elliptic algebras T: Sklyanin algebras and the quadric algebras defined by Van den Bergh in [VB3]. The algebras break into two cases, the first of which is when $T$ satisfies the hypotheses of Theorem 5.5. The other case is when gldim $T^{\circ}=2$. In the latter case we show that there is a Morita context between $T$ and a second elliptic algebra $T^{\prime}$ to which Theorem [5.5 can be applied. This is enough to prove that $T$ is also a minimal elliptic surface.

The formal definitions are as follows.

Example 6.1. The (quadratic) Sklyanin algebra is defined to be

$$
S:=S k l(a, b, c):=\mathbb{k}\left\{x_{1}, x_{2}, x_{3}\right\} /\left(a x_{i} x_{i+1}+b x_{i+1} x_{i}+c x_{i+2}^{2}: i \in \mathbb{Z}_{3}\right),
$$

where $[a: b: c] \in \mathbb{P}^{2} \backslash \Sigma$ for a (known) set $\Sigma$. Here, $S$ contains a canonical central element $g \in S_{3}$ such that $S / g S \cong B(E, \mathcal{L}, \sigma)$ for an elliptic curve $E$ with a line bundle $\mathcal{L}$ of degree 3 . In this paper we assume that $|\sigma|=\infty$, from which it follows that the 3 -Veronese $\operatorname{ring} T=S^{(3)}$ is an elliptic algebra, which we sometimes call the Sklyanin elliptic algebra. See, for example, [ATV1, Theorem 6.8(1)] for the details.

Example 6.2. The cubic Sklyanin algebra is defined as

$$
S^{\prime}:=S k l^{\prime}(a, b, c)=\mathbb{k}\left\{x_{1}, x_{2}\right\} /\left(a\left(x_{i+1}^{2} x_{i}+x_{i}^{2} x_{i+1}\right)+b x_{i+1} x_{i} x_{i+1}+c x_{i}^{3}: i \in \mathbb{Z}_{2}\right),
$$


for $[a: b: c] \in \mathbb{P}^{2} \backslash \Sigma$ for a known set $\Sigma$. Then $S^{\prime}$ contains a central element $g \in S_{4}^{\prime}$ so that $S^{\prime} / g S^{\prime} \cong B\left(E, \mathcal{L}^{\prime}, \sigma\right)$ for an elliptic curve $E$ with a line bundle $\mathcal{L}^{\prime}$ of degree 2. As above, we assume that $|\sigma|=\infty$, and so $\left(S^{\prime}\right)^{(4)}$ is an elliptic algebra. Details are in ATV1] as above. We will not need to treat this case separately, since the Veronese ring $\left(S^{\prime}\right)^{(2)}$ is a noncommutative quadric in the sense of the next example (this is implicit in VB3] and proven explicitly in [DL, Theorem 7.1]).

Example 6.3. Assume now that char $\mathbb{k}=0$. For the purposes of this paper we define noncommutative quadrics as follows. First, let $\mathrm{Skl}_{4}$ denote a 4-dimensional Sklyanin algebra; thus $\mathrm{Skl}_{4}$ is the $\mathbb{k}$-algebra with 4 generators $x_{0}, \ldots, x_{3}$ and the 6 relations

$$
x_{0} x_{i}-x_{i} x_{0}=\alpha_{i}\left(x_{i+1} x_{i+2}+x_{i+2} x_{i+1}\right), \quad x_{0} x_{i}+x_{i} x_{0}=x_{i+1} x_{i+2}-x_{i+2} x_{i+1},
$$

where the subscripts are taken to be $\{1,2,3\} \bmod 3$ and the $\alpha_{i}$ satisfy $\alpha_{1} \alpha_{2} \alpha_{3}+\alpha_{1}+\alpha_{2}+\alpha_{3}=0$ and $\left\{\alpha_{i}\right\} \cap\{0, \pm 1\}=\varnothing$. Significant properties from [SSf] are that $S=\mathrm{Skl}_{4}$ is a noetherian Artin-Schelter regular domain of dimension 4 , as defined in [SSf], with the Hilbert series $(1-t)^{-4}$ of a polynomial ring in 4 variables. Moreover, $S$ has a two-dimensional space of central homogeneous elements $V \subset S_{2}$. The factor $\operatorname{ring} S / S V$ is isomorphic to a twisted homogeneous coordinate $\operatorname{ring} B(E, \mathcal{L}, \sigma)$, where $E$ is a smooth elliptic curve and $\sigma$ is an automorphism given by translation by a point also denoted $\sigma \in E$. However, $\mathcal{L}$ is now a line bundle of degree 4 . For the purposes of this paper we again assume that $|\sigma|=\infty$.

Given a non-zero element $\Omega \in V$ we define the Van den Bergh quadric $Q_{V d B}:=Q_{V d B}(\Omega):=S / S \Omega$. As explained, for example in $[\mathrm{SmV}$ this is a domain which is Artin-Schelter Gorenstein in the sense of [ASc]. Fixing a basis element $g$ for the image of $V$ in $Q_{V d B}$ ensures that $T:=Q_{V d B}{ }^{(2)}$ is an elliptic algebra, which we term a quadric elliptic algebra.

The structure of $Q_{V d B}$ depends upon the choice of $\Omega$ and so we refine our notation as follows. We use the notation from $[\mathrm{SmV},(10.3)]$, where the reader is referred for more details. First identify $E \subset \mathbb{P}\left(S_{1}^{*}\right)$. Given two points $p, q \in E$ we can define a left $S$-line module $L(\overline{p q}):=S / S W$, where $W \subset S_{1}$ consists of the forms vanishing on the line $\overline{p q} \subset \mathbb{P}\left(S_{1}^{*}\right)$. It is known that, up to scalar multiple, there exists a unique $\Omega \in V$ vanishing on $L(\overline{p q})$. In fact, $\Omega$ depends only on the sum $r=p+q$ under the group law on $E$, so we write $\Omega=\Omega(r)$. Finally, we define $Q_{V d B}(r):=S / S \Omega(r)$. As noted in $[\mathrm{SmV},(10.3)], Q_{V d B}(r)=Q_{V d B}(-r-2 \sigma)$.

Remark 6.4. The characteristic zero hypothesis is only needed in Example 6.3 because this hypothesis is assumed in the results we cite from [LS, VB1], and we conjecture that all the results hold over arbitrary fields. Certainly our results on quadratic Sklyanin algebras are characteristic-free, and one can give characteristicfree proofs for the results on cubic Sklyanin algebras.

We remark that in VB3], Van den Bergh actually defines his quadrics as certain categories deforming the category of quasicoherent sheaves on a commutative quadric surface. It is only after his classification that one can identify his "elliptic" quadrics as qgr- $R$ for factors $R$ of $\mathrm{Skl}_{4}$. Since this relationship is not relevant to the present paper, we refer the interested reader to [VB3] and [StV] Sections 11 and 12] for the details. 
These quadrics include, but are much more general than, the noncommutative analogue of $\mathbb{P}^{1} \times \mathbb{P}^{1}$ (see VB3, Introduction]) as well as a sort of noncommutative analogue of a Hirzebruch surface $F_{2}$ (see [SmV], Section 10.5]).

Much is known about the Van den Bergh quadrics, and we begin by collecting some of those known facts. We start with another definition.

Definition 6.5. If $T$ is a cg noetherian algebra, then qgr- $T$ is smooth if it has finite homological dimension. By [RSS4, Lemma 6.8], if $T$ is an elliptic algebra, this is equivalent to asserting that gldim $T^{\circ}<\infty$.

Lemma 6.6. Let $Q:=Q_{V d B}(r)=S / \Omega(r)$ for some $r \in E$, and let $E_{2}$ denote the points of order 2 in $E$. Then:

(1) qgr- $Q$ is smooth if and only if $r+\sigma \notin E_{2}$.

(2) Assume that $r+\sigma \notin E_{2}$. Then either $Q^{\circ}$ is simple hereditary or $\operatorname{gldim} Q^{\circ}=2$. In the latter case $Q^{\circ}$ has, up to isomorphism, a unique finite dimensional simple module, say of dimension d. This occurs if and only if $r=\omega+n \sigma$ for some $\omega \in E_{2}, n \in \mathbb{Z} \backslash\{-1\}$, with $d=|n+1|$.

(3) If $r+\sigma \in E_{2}$, then $Q^{\circ}$ is simple of infinite global homological dimension.

(4) All the above results also hold for $Q=Q_{V d B}(r)^{(2)}$.

Proof. (1) This is $\mathrm{SmV}$, Theorem 10.2].

(2) By Lemma 4.3, $Q^{\circ}$ is $\mathrm{CM}$ of finite injective dimension and Gelfand-Kirillov dimension 2, for any choice of $r$. As such, if qgr- $Q$ is smooth then, necessarily, either $Q^{\circ}$ is simple or gldim $Q^{\circ}=2$, in which case $Q^{\circ}$ has at least one finite dimensional simple module. So we need to identify when the latter happens.

Recall that a fat point over $Q$ (or $S$ ) is a 1-critical graded $Q$-module $M$. As such, there exists $d$ such that $\operatorname{dim}_{\mathbb{k}} M_{n}=d$, for $n \gg 0$, called the multiplicity of $M$. Note that if $M$ is a 1-critical $S$-module, then either $M$ is a $g$-torsion point module (and hence a $B$-module) or $M$ is killed by at most one $\Omega \in V$, up to scalar multiples.

If $M$ is a $g$-torsionfree point module, then [SSf] Proposition 2.4] implies that $M$ is one of 4 exceptional point modules and, by [LS, Theorem 5.7] this implies that $r \in\{\omega, \omega-2 \sigma\}$ for some $\omega \in E_{2}$. On the other hand, if $M$ is a fat point of multiplicity $d>1$, then $r \in\{\omega-1 \pm d \sigma\}$ by [SSn, Proposition 4.4]. Moreover, by [SSn, Remark p.84] if $r \in\{\omega-1 \pm d \sigma\}$ for $d \geqslant 1$, there is only one fat point in qgr- $Q$. By [RSS1, Lemma 2.1], $Q^{\circ} \cong Q^{(2)} /(g-1)$. Thus $Q^{\circ}$ then has exactly one finite dimensional, simple module $M^{\circ}$ and, moreover, $\operatorname{dim}_{\mathbb{k}} M^{\circ}=d$.

(3) The proof is similar to that of Lemma 4.4. Assume that $r+\sigma \in E_{2}$. By (1) gldim $Q^{\circ}=\infty$ and, by (2), it cannot have any finite dimensional modules. Suppose, however, that $Q^{\circ}$ is not a simple ring; thus it has a proper, nonzero prime factor ring $A=Q^{\circ} / I$. By [KL, Proposition 3.15], GKdim $A \leqslant 1$ and so, by [SW] and [KL, Corollary 10.9], $A$ has a nonzero finite dimensional module, a contradiction. Thus $Q^{\circ}$ is simple.

(4) This follows immediately from the fact that $\operatorname{qgr}-Q=\operatorname{qgr}-Q^{(2)}$ (see for example [AS, Proposition 6.2]) and $Q^{\circ}=\left(Q^{(2)}\right)^{\circ}$. 
If $r^{\prime}=r+n \sigma$ for some $n \in \mathbb{Z}$ then Van den Bergh shows there is a Morita context between $Q_{V d B}(r)$ and $Q_{V d B}\left(r^{\prime}\right)$. In the next result, we show that if $n=1$, this Morita context is given by a line ideal: a right ideal $J$ of $Q=Q_{V d B}(r)$ so that $Q / J$ is a line module.

As a matter of notation, suppose that $A$ is a noetherian domain with division ring of fractions $\operatorname{Fr}(A)$ and a finitely generated fractional right ideal $J$. Then we will identify $J^{*}=\operatorname{Hom}_{A}(J, A)$ with $\{\theta \in \operatorname{Fr}(A): \theta J \subseteq A\}$ and $\operatorname{End}_{A}(J)$ with $\{\theta \in \operatorname{Fr}(A): \theta J \subseteq J\}$, with similar conventions for left modules. The following well-known lemma will be used frequently.

Lemma 6.7. Let $A$ and $A^{\prime}$ be noetherian maximal orders with the same division ring of fractions $\operatorname{Fr}(A)$ and suppose that there is an $\left(A, A^{\prime}\right)$-bimodule $M$ contained in $\operatorname{Fr}(A)$ and finitely generated on both sides. Then;

(1) $A^{\prime}=\operatorname{End}_{A}(M)=\operatorname{End}_{A}\left(M^{* *}\right)$;

(2) $\operatorname{Hom}_{A}(M, A)=\operatorname{Hom}_{A^{\prime}}\left(M, A^{\prime}\right)$, so the notation $M^{*}$ is unambiguous.

Remark 6.8. All the rings that concern us, in particular Sklyanin elliptic algebras, quadric elliptic algebras as well as the Sklyanin and quadric algebras themselves are maximal orders by [Rg, Theorem 6.7] and so the lemma applies to them.

Proof. (1) By hypothesis $A$ and $A^{\prime}$ are equivalent orders in the sense that there exists non-zero elements $a, b, c, d \in \operatorname{Fr}(A)$ such that $a A^{\prime} b \subseteq A$ and $c A d \subseteq A^{\prime}$. Similarly, $A$ and $\operatorname{End}_{A}(M)$ are equivalent orders. Moreover $A^{\prime} \subseteq \operatorname{End}_{A}(M)$. Now apply [RSS2, Lemma 6.2].

(2) Note that $M^{*}=\operatorname{Hom}_{A}(M, A)$ satisfies $M M^{*} \subseteq A^{\prime}=\operatorname{End}_{A}(M)$ and so $M^{*} \subseteq \operatorname{Hom}_{A^{\prime}}\left(M, A^{\prime}\right)$. So $M^{*}=\operatorname{Hom}_{A^{\prime}}\left(M, A^{\prime}\right)$ by symmetry.

Lemma 6.9. Let $Q:=Q_{V d B}(r)$ be a Van den Bergh quadric, and let $r^{\prime}=r+\sigma$. Then there is a right ideal $J$ of $Q$ so that $\operatorname{End}_{Q}(J) \cong Q_{V d B}\left(r^{\prime}\right)$ and $\operatorname{End}_{Q_{V d B}\left(r^{\prime}\right)}(J) \cong Q$. Thus $J$ is a $\left(Q_{V d B}\left(r^{\prime}\right), Q\right)$-bimodule. Further, we may choose $J$ to be a line ideal that is MCM.

Proof. Set $Q^{\prime}:=Q_{V d B}\left(r^{\prime}\right)$ and $A:=\mathrm{Skl}_{4}$. By [VB1, (7.12) and Lemma 7.4.3], there is a $\left(Q^{\prime}, Q\right)$-bimodule $J$ with

$$
\text { hilb } J=\operatorname{hilb} Q-1 /(1-t)^{2}+H(t) \quad \text { for some some Laurent polynomial } H(t)
$$

so that $\operatorname{End}_{Q}(J) \cong Q^{\prime}$. Technically, $J$ is only defined as an element of qgr- $Q$, so we take $J$ to be some representative in $\operatorname{gr}-Q$ of this equivalence class. In particular, from the proof and notation of [VB1, Lemma 7.4.3] we can take

$$
J:=\bigoplus_{m} \Gamma\left(\mathbb{P}^{1}, \mathcal{O}_{\mathbb{P}^{1}}(-1) \bigotimes_{\mathbb{P}^{1}}\left(\mathcal{B}_{z+2 \sigma}\right)_{m}\right) \subseteq \bigoplus_{m} \Gamma\left(\mathbb{P}^{1},\left(\mathcal{B}_{z+2 \sigma}\right)_{m}\right)=Q
$$

so $J$ is a right ideal of $Q$. Next, as $Q^{\prime}$ is a maximal order by Remark 6.8, it follows from Lemma 6.7 that $Q^{\prime}=\operatorname{End}_{Q}\left(J^{* *}\right)$, and so we can replace $J$ by $J^{* *}$. Necessarily, $\operatorname{GKdim}(Q / J)=2$ still holds and $L=Q / J$ still has multiplicity one. However, by the CM condition, $L$ now has no submodules of GKdim $\leqslant 1$. 
Now consider $L$ as an $A$-module. By the CM condition for $A$, we have $j_{A}(L)=\operatorname{GKdim} A-\operatorname{GKdim} L=2$. Thus, by [LS, Proposition 2.1(f)], both $L^{\vee}:=\operatorname{Ext}_{A}^{2}(L, A)$ and $L^{\vee \vee}:=\operatorname{Ext}_{A}^{2}\left(\operatorname{Ext}_{A}^{2}(L, A), A\right)$ are CM over $A$ with GK-dimension 2. Moreover, [LS, Proposition 2.1(d)] implies that the natural map $\psi: L \rightarrow L^{\vee \vee}$ has $\operatorname{GKdim}(\operatorname{Ker}(\psi)) \leqslant 1$ and $\operatorname{GKdim}(\operatorname{Coker}(\psi)) \leqslant 0$. Hence $\operatorname{Ker}(\psi)=0$ and $L^{\vee \vee}$ also has multiplicity 1. By [LS, Proposition 2.12] $L^{\vee \vee}$ is therefore a line module and, in particular, is cyclic. Since $L^{\vee \vee}$ only differs from $L$ by a finite dimensional module, and has a prime annihilator [LS, Theorem 6.3], it follows that $L^{\vee \vee}$ is a $Q$-module, say $L^{\vee \vee}=Q / J^{\prime}$. Moreover, $J^{\prime} \supseteq J Q_{\geqslant m}$ for some $m$. Since neither $Q / J^{\prime}$ nor $Q / J$ has a non-zero, finite dimensional submodule, it follows that $J=J^{\prime}$. By [SmV, Proposition 7.2] this also ensures that $J$ is MCM, and so $J$ is the right ideal we seek.

Finally, the assertion that $\operatorname{End}_{Q^{\prime}}(J) \cong Q$ follows from Lemma 6.7 and Remark 6.8

Lemma 6.10. Keep the set-up from Lemma 6.9 and let $J$ be the $\left(Q^{\prime}, Q\right)$-bimodule constructed there. Then $J=J_{1} Q=Q^{\prime} J_{1}$.

Proof. For $J_{Q}$, the result follows from [RSS4, Lemma 5.6(2)], but a little more work is needed on the left.

Let $B:=Q / Q g=B(E, \mathcal{N}, \sigma)$ and $B^{\prime}:=Q^{\prime} / Q^{\prime} g=B\left(E, \mathcal{N}^{\prime}, \sigma\right)$ for the appropriate invertible sheaves $\mathcal{N}, \mathcal{N}^{\prime}$ of degree 4 on $E$. By [RSS4, Lemma 5.6(2) and Notation 2.2], $\bar{J}$ is a saturated right $B$-module, in the sense that it has no finite dimensional extensions. Moreover it is a torsion-free $B$-module as $J$ is $g$-divisible. As $g$ acts centrally, $\bar{J}$ is a therefore a torsion-free left $B^{\prime}$-module. We first prove that $\bar{J}$ is also saturated on the left. By Le, (4.6.6) and Remark 5.8(4)], $X:=\operatorname{Hom}_{B^{\prime}}\left(\operatorname{Hom}_{B^{\prime}}\left(\bar{J}, B^{\prime}\right), B^{\prime}\right)$ is the maximal essential extension of $\bar{J}$ by finite dimensional left $B^{\prime}$-modules. Moreover, $X / \bar{J}$ is finite dimensional, say with $K X \subset \bar{J}$ for $K:=B_{\geqslant m}^{\prime}$. Clearly $X \subset Q_{\mathrm{gr}}\left(B^{\prime}\right)=Q_{\mathrm{gr}}(B)$ and so if $y \in B$ then $K X y \subseteq \bar{J} y \subseteq \bar{J}$. Hence $X y+\bar{J}$ is a finite dimensional extension of $\bar{J}$ and so $X y \subseteq X$. Thus $X$ is a right $B$-module which, as $\bar{J}_{B}$ is saturated, implies that $X=\bar{J}$; in other words, $\bar{J}$ is saturated as a left $B^{\prime}$-module. Therefore, by [AV, Theorem 1.3], $\bar{J}=\oplus_{n \geqslant 0} H^{0}\left(E, \mathcal{N}_{n}^{\prime} \otimes \mathcal{O}(\mathbf{p})^{\sigma^{n}}\right)$, for some divisor $\mathbf{p}$. Since $\bar{J}_{Q}$ is a line ideal, $\operatorname{dim} \bar{J}_{1}=\operatorname{dim} Q_{1}-2=2$, and so $\operatorname{deg} \mathbf{p}=-2$, by Riemann-Roch. Therefore, $\mathrm{Rg}$, Lemma 3.1] implies that $\bar{J}$ is generated in degree 1 as a left $B^{\prime}$-module. By the graded Nakayama lemma, $J$ is also generated in degree 1 as a left $Q^{\prime}$-module.

We immediately obtain:

Corollary 6.11. Let $Q:=Q_{V d B}(r)$ be a Van den Bergh quadric, and let $r^{\prime}=r+n \sigma$ for some $n \in \mathbb{Z}$. Then there is a graded rank one torsionfree right $Q$-module $M=M_{r^{\prime}, r}$ so that $Q^{\prime}:=Q_{V d B}\left(r^{\prime}\right) \cong \operatorname{End}_{Q}(M)$, while $\operatorname{End}_{Q^{\prime}}(M)=Q$ and both $Q_{Q^{\prime}} M$ and $M_{Q}$ are finitely generated.

Proof. Take $M$ to be the appropriate product of the bimodules $J$ constructed by Lemma 6.9. Again, Lemma 6.7 and Remark 6.8 ensures that $\operatorname{End}_{Q^{\prime}}(M)=Q$.

Proposition 6.12. Let $T:=Q_{V d B}{ }^{(2)}$ be the 2-Veronese of a Van den Bergh quadric. If $T^{\circ}$ is simple, then either $T^{\circ}$ is hereditary, or $T^{\circ}$ has an $A_{1}$ singularity, as in Definition 5.1 .

If $T=S k l^{(3)}$ or $T=S k l^{(4)}$, then $T^{\circ}$ is simple and hereditary. 
Proof. By [ATV2, Theorem 7.3] localised Sklyanin elliptic algebras are simple (this uses our standing hypothesis that $|\sigma|=\infty)$. If $T=S k l^{(3)}$ then $T^{\circ}$ is hereditary by [Aj, Proposition 2.18]. The same proof works for $T=S k l^{(4)}$. If $T=Q_{V d B}{ }^{(2)}$, then the result is clear by Lemma 6.6, unless $T^{\circ}$ has infinite global dimension. By Lemma 6.6 this happens only if $T=Q^{(2)}$, where $Q=Q_{V d B}(\omega-\sigma)$ for some $\omega \in E_{2}$.

So, fix such a $Q:=Q_{V d B}(\omega-\sigma)$, set $Q^{\prime}:=Q_{V d B}(\omega)$ and let $J$ be the right ideal of $Q$ constructed in

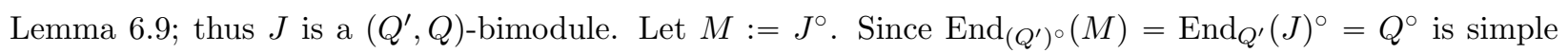
by Lemma 6.6(3), $M$ is a projective left $\left(Q^{\prime}\right)^{\circ}$-module by the Dual Basis Lemma. As $\operatorname{gldim}\left(Q^{\prime}\right)^{\circ}=2$ and $\operatorname{gldim} Q^{\circ}=\infty$, certainly $\left(Q^{\prime}\right)^{\circ}$ and $Q^{\circ}$ are not Morita equivalent. Thus $\left(Q^{\prime}\right)^{\circ} M$ is not a generator; equivalently $M M^{*} \neq\left(Q^{\prime}\right)^{\circ}$ and so $Q^{\prime} / J J^{*}$ is infinite-dimensional. We claim that $Q^{\prime} / J J^{*} \cong \mathbb{k}[g]$.

By Lemma 6.10, $Q^{\prime} J$ and $J_{Q}$ are generated by $J_{1}$, which has dimension 2. In particular, $J[1]$ is in the set $\mathbb{M}$ of right MCM $Q$-modules defined in $[\mathrm{SmV}, 5.3]$. Thus by $[\mathrm{SmV}, \operatorname{Lemma} 7.1], \operatorname{dim}\left(J^{*}\right)_{0}=2$, say with basis $\{a, b\}$. If $J a=J b$, then $J a b^{-1}=J$ and so $a b^{-1} \in \operatorname{End}_{Q^{\prime}}(J)_{0}=Q_{0}=\mathbb{k}$, which is impossible. Thus $J a \neq J b$. Since $J=Q^{\prime} J_{1}$ it follows that $\operatorname{dim}\left(J J^{*}\right)_{1} \geqslant 3$; equivalently $\operatorname{codim}\left(J J^{*}\right)_{1} \leqslant 1$. We note also that $J_{<0}^{*}=0$ as $J$ is not cyclic.

By [RSS4, Lemma 5.6S(2)], $\bar{J}$ is saturated and so, as in the proof of Lemma 6.10, we may write $B:=$ $Q / Q g=B(E, \mathcal{N}, \sigma)$, and $\bar{J}=\bigoplus_{n \geqslant 0} H^{0}\left(E, \mathcal{O}(\mathbf{q}) \otimes \mathcal{N}_{n}\right)$, for some invertible sheaf $\mathcal{N}$ and divisor q with $\operatorname{deg} \mathbf{q}=-2$. By [RSS2, Lemma 6.14]

$$
\overline{J^{*}} \subseteq(\bar{J})^{*}=\bigoplus_{n \geqslant 0} H^{0}\left(E, \mathcal{N}_{n} \otimes \mathcal{O}(-\mathbf{q})^{\sigma^{n}}\right)
$$

By [RSS4, Lemma 5.6] and [RSS2, Lemma 2.12], $J$ and hence $J^{*}$ are $g$-divisible. Thus $\operatorname{dim} J_{0}^{*}=\operatorname{dim} \bar{J}_{0}=2$ by the last paragraph, while $\operatorname{dim}(\bar{J})_{0}^{*}=2$ by (6.13) and Riemann-Roch. Thus, $\bar{J}_{0}^{*}=(\bar{J})_{0}^{*}$. By $\operatorname{Rg}$, Lemma 3.1] and (6.13), $(\bar{J})^{*}$ is generated in degree zero and so $\overline{J^{*}}=(\bar{J})^{*}$. By [Rg, Lemma 3.1], again, $\left(\overline{J J^{*}}\right)_{n}=B_{n}$ for $n \geqslant 2$. Since $\left(J J^{*}\right)_{n+2} \supseteq g\left(J J^{*}\right)_{n}$, it follows that $\operatorname{codim}\left(J J^{*}\right)_{n+2} \leqslant \operatorname{codim}\left(J J^{*}\right)_{n}$ for all $n \in \mathbb{N}$. This codimension is bounded by 1 in degrees 0 and 1 , so $\operatorname{codim}\left(J J^{*}\right)_{n} \leqslant 1$ for all $n \geqslant 0$. Conversely, as $\operatorname{dim} Q^{\prime} / J J^{*}=\infty$, clearly $\left(J J^{*}\right)_{n} \neq Q_{n}^{\prime}$ holds for all $n \geqslant 0$. Thus $P=Q^{\prime} / J J^{*}$ is a point module, from which it follows that $P$ is $g$-torsionfree since $P^{\circ} \neq 0$. Thus $P \cong \mathbb{k}[g]$, as claimed.

Since $P=\operatorname{End}_{Q}(J) / J J^{*} \cong \mathbb{k}[g]$, the stable endomorphism ring of $M=J^{\circ}$ is

$$
\text { End }_{Q^{\circ}}(M)=\left(Q^{\prime}\right)^{\circ} / M M^{*}=\mathbb{k} .
$$

We are now ready to apply Proposition 5.8 to $A=Q^{\circ}$. Bt the second paragraph of the proof, gldim $Q^{\circ}=\infty$ while $\left(Q^{\prime}\right)^{\circ}=\operatorname{End}_{Q^{\circ}}(M)$ has gldim $\left(\left(Q^{\prime}\right)^{\circ}\right)=2$. Moreover, as $Q^{\circ}$ is simple, $M$ is automatically a generator. Finally, $\operatorname{inj\operatorname {dim}}\left(Q^{\circ}\right)=1$ and $M$ is a MCM $Q^{\circ}$-module by Lemma 4.3. By Proposition 5.8 and the last display, $Q^{\circ}$ therefore has an $A_{1}$ singularity.

We next note that these elliptic algebras never have line modules. 
Lemma 6.14. Let $A$ be a cg noetherian algebra of finite global dimension such that hilb $A=(1-t)^{-n}$ for some $n \geqslant 2$. Then for $d>1$, the Veronese ring $A^{(d)}$ has no linear modules other than point modules.

Proof. Let $L$ be a finitely generated graded $A^{(d)}$-module with Hilbert series $(1-t)^{-p}$. Let $\widetilde{L}:=L \otimes_{A^{(d)}} A$, and note that $L=\widetilde{L}^{(d)}$. Let $P_{\bullet} \rightarrow \widetilde{L} \rightarrow 0$ be a finite graded free resolution of $\widetilde{L}$. Thus, each $P_{j} \cong$ $\bigoplus_{i=1}^{\ell_{j}} A\left[-a_{i j}\right]$ for some natural numbers $\ell_{j}, a_{i j}$ and so hilb $\widetilde{L}=F(t) /(1-t)^{n}$ for $F=\sum_{j}(-1)^{j} \sum_{i} t^{a_{i j}}$. Since $p=\operatorname{GKdim}(\widetilde{L})=\operatorname{GKdim} L$, we can cancel common factors of $(1-t)$ from this equation to obtain a polynomial $G \in \mathbb{Z}[t]$, with $G(1) \neq 0$, such that hilb $\widetilde{L}=G(t) /(1-t)^{p}$. Rewriting $G$ as an integer polynomial in $1-t$ gives

$$
\text { hilb } \widetilde{L}=H(t)+\sum_{k=1}^{p} c_{k} /(1-t)^{k},
$$

where $H \in \mathbb{Z}[t]$ and the $c_{k} \in \mathbb{Z}$. Thus there exists $f(t) \in \mathbb{Q}[t]$ with leading term $c_{p} t^{p-1} /(p-1)$ ! with $\operatorname{dim} \widetilde{L}_{n}=f(n)$ for $n \gg 0$. Thus $\operatorname{dim} L_{n}=\operatorname{dim} \widetilde{L}_{d n}=f(d n)$ for $n \gg 0$. But $\operatorname{dim} L_{n}=\left(\begin{array}{c}n+p-1 \\ p-1\end{array}\right)$ by hypothesis. Thus, taking leading terms and multiplying by $(p-1)$ ! gives $c_{p}(d n)^{p-1}=n^{p-1}$ for $n \gg 0$. As $d>1$ and $c_{p} \in \mathbb{Z}$ this is only possible if $p=1$.

Corollary 6.15. If $T=S k l^{(3)}$ or $T=S k l^{(4)}$ or $T=Q_{V d B}^{(2)}$, then $T$ has no line modules.

Proof. For $T=S k l^{(3)}$ the result is immediate from Lemma 6.14. For the other cases use the fact that $Q_{V d B}$ (and hence $T=S k l^{(2)}$ ) is, by definition, a factor of the Sklyanin algebra $A=\operatorname{Skl}_{4}$ (see Example 6.3).

Putting together the results of this section gives our main theorem.

Theorem 6.16. Let $T=Q_{V d B}{ }^{(2)}$ or $T=S k l^{(3)}$ or $T=S k l^{(4)}$. Then $T$ is a minimal elliptic surface.

Indeed, suppose that $T \subseteq R \subset T_{(g)}$ is a finitely graded overring such that either $R$ is noetherian or $\widehat{R}$ is finitely graded. Then $R=T$.

Proof. Suppose first that $T^{\circ}$ is simple. By Proposition 6.12 and Corollary6.15, $T \subseteq R$ satisfies the hypotheses of Theorem [5.5] and so the result follows from that theorem.

Now suppose that $T^{\circ}$ is not simple. Then Proposition 6.12 and Lemma 6.6 imply that $T=Q^{(2)}$, where $Q:=Q_{V d B}(\omega+k \sigma)$ for some $\omega \in E_{2}$ and $k \in \mathbb{N}$. Let $T_{\infty}:=\left(Q_{V d B}(\omega-\sigma)\right)^{(2)}$. Let $M$ denote the $\left(\left(Q_{V d B}(\omega-\sigma), Q\right)\right.$-bimodule constructed by Corollary 6.11 and set $N=M^{(2)}$. Clearly, $N$ is a $\left(T_{\infty}, T\right)$ bimodule that is finitely generated on both side and contained in $Q_{g r}(T)$. It follows from Lemma 6.7 that $K=N^{* *}$ is a $\left(T_{\infty}, T\right)$-bimodule contained in $Q_{g r}(T)=Q_{g r}\left(T_{\infty}\right)$ such that $\operatorname{End}_{T}(K)=T_{\infty}$ and, conversely, $\operatorname{End}_{T_{\infty}}(K)=T$. Suppose that the theorem is false, say for the overring $T \varsubsetneqq R \subset T_{(g)}$. If $R$ is noetherian, then Lemma 3.9 implies that $\widehat{R}$ is a finitely generated $R$-module and hence is finitely graded. Thus, in all cases, we can replace $R$ by $\widehat{R}$ and assume that $R$ is finitely graded with $R=\widehat{R}$. We aim for a contradiction.

By Lemma 6.7 $K^{*}=\operatorname{Hom}_{T}(K, T)=\operatorname{Hom}_{T_{\infty}}\left(K, T_{\infty}\right)$. Proposition 5.8 Set $\mathfrak{m}=K K^{*}$; this is an ideal of $T_{\infty}$ which, since $T_{\infty}^{\circ}$ is simple, contains $g^{n}$ for some $n \geqslant 1$. Let $U:=T_{\infty}+K R K^{*}$, which is certainly a finitely graded ring with $T_{\infty} \subseteq U \subseteq\left(T_{\infty}\right)_{(g)}=T_{(g)}$. If $v \in \widehat{U}$, then $g^{m} v \in U$ for some $m \geqslant 1$ and so $g^{m}\left(K^{*} v K\right) \subseteq$ 
$K^{*} U K \subseteq R=\widehat{R}$. Thus $K^{*} v K \subseteq R$ and so $\mathfrak{m} v \mathfrak{m}=K K^{*} v K K^{*} \subseteq U$. Thus $g^{2 n} \widehat{U} \subseteq \mathfrak{m} \widehat{U} \mathfrak{m} \subseteq U$. Thus $\widehat{U}$ is finitely graded. By Theorem 5.5 this forces $\widehat{U}=T_{\infty}$ and so

$$
\left(K^{*} K\right) R\left(K^{*} K\right) \subseteq K^{*} T_{\infty} K=K^{*} K \subseteq T
$$

Therefore, $T$ and $R$ are equivalent orders which, by [Rg, Theorem 6.7], implies that $T=R$.

Remark 6.17. Suppose that $T$ is one of the algebras in the Theorem, and that $T \varsubsetneqq R=\widehat{R} \subseteq T_{(g)}$ is a $g$-divisible graded overring. Then we can further apply Theorem 5.5 to conclude that $\operatorname{dim} R_{n}=\infty$ for some $n \geqslant 0$. If $T^{\circ}$ is simple one can even show that $\operatorname{dim} R_{0}=\infty$ and we conjecture that this holds without the assumption of simplicity.

The conclusion of Theorem 6.16 also extends to Sklyanin algebras and Van den Bergh's quadrics themselves.

Corollary 6.18. Let $S=S k l$ or $S=S k l^{\prime}$ or $S=Q_{V d B}$. Then $S$ is a minimal surface in the sense that if $S \subseteq U \subset S_{(g)}$ for some cg noetherian ring $U$ then $S=U$.

Proof. Fix such a ring $U$ and pick $d$ so that $T=S^{(d)}$ is an elliptic algebra. Then $T \subseteq U^{(d)} \subset T_{(g)}$. By [AS, Lemma 4.10] $U^{(d)}$ is noetherian and cg and so, by Theorem 6.16. $U^{(d)}=T$. If $U \neq S$, pick $v \in U_{n d+r} \backslash S_{n d+r}$ for some $n$ and $0<r<d$. Then $v S_{1}^{d-r} \subseteq U^{(d)}=S^{(d)} \subset S$. Since $S$ is generated in degree 1 it follows that $v S_{\geqslant d} \subseteq S$ and hence that $(v S+S) / S$ is finite dimensional. By the CM property for $S$ (see [Le, (4.6.6) and Remark 5.8(4)]) this forces $v \in S$ and hence $U=S$.

\section{OVERRINGS NOT CONTAINED IN $T_{(g)}$}

Let $T$ be one of the algebras from Theorem 6.16, or indeed any minimal elliptic surface; thus by definition there is no noetherian cg ring $T \varsubsetneqq U \subset T_{(g)}$. There do, however, exist cg noetherian overrings of $T$ inside $Q_{g r}(T)$. Indeed, for any integer $n \geqslant 2$ one has $T \subseteq R:=\mathbb{k}\left\langle T_{n} g^{1-n}\right\rangle$. This is of course a rather "cheap" counterexample since after a change of grading, $\mathbb{k}\left\langle T_{n} g^{1-n}\right\rangle \cong T^{(n)}$ under the homomorphism $x g^{1-n} \mapsto x$ for all $x \in T_{n}$. As we show in this section the possible cg noetherian overrings of $T$ are tightly constrained, and are all quite similar to the "cheap" example above. We do, however, need the technical assumption that the overring is a maximal order.

Theorem 7.1. (1) Let $T$ be a minimal elliptic surface and let $T \subseteq R \subset Q_{g r}(T)$ be a cg noetherian overring of $T$ that is a maximal order in $Q_{g r}(T)$. Then for each $n$ there is $\ell \geqslant n$ so that $R_{n}=T_{\ell} g^{n-\ell}$, and there exist integers $M \geqslant N \geqslant 1$ so that $R^{(N)}=\mathbb{k}\left\langle T_{M} g^{N-M}\right\rangle \cong T^{(M)}$.

(2) Similarly, let $S=Q_{V d B}$ or $S=S k l$ or $S=S k l^{\prime}$ and let $d=\operatorname{deg} g$. Let $S \subseteq A \subset Q_{g r}(S)$ be a cg noetherian overring of $S$ that is a maximal order in $Q_{g r}(S)$. Then for all $n$, there is $\ell \in \mathbb{Z}$ so that $A_{n}=g^{\ell} S_{n-d \ell}$, and there are integers $N, M \geqslant 1$ so that $A^{(N)} \cong S^{(M)}$. 
Proof. (1) For $j \in \mathbb{N}$, define

$$
\phi_{j}: Q_{g r}(T) \rightarrow\left(Q_{g r}(T)\right)^{(j+1)} \quad \text { by } \phi_{j}(f)=f g^{j n} \text { for all } f \in Q_{g r}(T)_{n} .
$$

Since $g$ is central, $\phi_{j}$ is a $\mathbb{k}$-algebra homomorphism. Since $R$ is cg, and hence a finitely generated algebra, there exists $j \geqslant 0$ with $\phi_{j}(R) \subset T_{(g)}$. Let $U:=\phi_{j}(R)$. Define $h: \mathbb{N} \rightarrow \mathbb{N} \cup\{\infty\}$ by

$$
h(n):=\max \left\{i: U_{n} \subseteq g^{i} T_{(g)}\right\}, \text { with } h(n):=\infty \text { if } U_{n}=0 .
$$

We begin by following the proof of [RSS2, Proposition 9.1], although as our notation is slightly different we give most of the details.

Let $U$ be generated in degrees $\leqslant r$, so $U_{n}=\sum_{i=1}^{r} U_{i} U_{n-i}$ for all $n>r$. As in [RSS2], the fact that $g T_{(g)}$ is a completely prime ideal implies that $h(n)+h(m) \geqslant h(n+m)$ for all $n, m \in \mathbb{N}$ and, moreover, that

$$
h(n)=\min \{h(n-i)+h(i): 1 \leqslant i \leqslant r\} \quad \text { for } n>r,
$$

with the obvious convention if any of these numbers equals $\infty$. Now choose $1 \leqslant K \leqslant r$ so that $\lambda=h(K) / K$ is minimal. Applying induction to (7.3), we have $h(n) \geqslant \lambda n$ for all $n \in \mathbb{N}$, and this forces

$$
h(n K)=n h(K) \quad \text { for all } n \in \mathbb{N} .
$$

Since $U=\phi_{j}(R) \subseteq\left(T_{(g)}\right)^{(j+1)}$ and $U_{K} \neq 0$, the number $N:=K /(j+1)$ is an integer. Let $D:=h(K)$, and note that $D<K$, since if $D=K$ then $U_{K} \subseteq g^{K} T_{(g)}$, and so $R_{N} \subseteq g^{N} T_{(g)}$, contradicting $T_{N} \subseteq R_{N}$. Let $M=K-D$. By (7.4), $U_{n K} \subseteq g^{n D} T_{(g)}$ for all $n \in \mathbb{N}$. Thus the function $U_{n K} \rightarrow\left(T_{(g)}\right)_{n M}$ given by $x \mapsto x g^{-n D}$ is well-defined, and induces an injective vector space homomorphism $\theta: U^{(K)} \rightarrow T_{(g)}^{(M)}$ with $\theta\left(U^{(K)}\right) \nsubseteq \mathbb{k}+g T_{(g)}$. It is routine to see that $\theta$ is an algebra homomorphism.

Let $V:=\theta\left(U^{(K)}\right)$ and $Z:=\widehat{V[g]} \subset T_{(g)}$, recalling Notation 4.7. Since $T_{1} \subset R$, both $g^{j} T_{1}$ and $g^{K-1} T_{1}$ are contained in $U$. Thus $g^{M-1} T_{1}=\theta\left(g^{K-1} T_{1}\right) \subset V$, and in particular $g^{M} \in V$. By Lemma 3.9, $Z$ and $Z^{(M)}$ are finitely generated left and right $V$-modules and are thus cg noetherian. Further, as $g^{M-1} T_{1} \subseteq V$, we have $Z \supseteq T$ which, as $T$ is a minimal elliptic surface, forces $T=Z$. Thus $V=\theta\left(U^{(K)}\right) \subseteq T$ and so $U^{(K)} \subseteq T$.

We claim that in fact $U \subseteq T$. To see this, take $U_{n} \neq 0$. Since $R \supseteq T$, clearly $U_{n} \supseteq g^{a} T_{b}$ for some $a, b \geqslant 1$. Therefore, $g^{a(K-1)} U_{n} T_{b(K-1)}=U_{n}\left(g^{a} T_{b}\right)^{K-1} \subseteq U_{n K} \subseteq T$. As $T$ is $g$-divisible, it follows that $U_{n} T_{c} \subseteq T$ for $c=b(K-1)$. Hence $U_{n} T_{\geqslant c}=U_{n} T_{c} T \subseteq T$ and so $\left(U_{n} T+T\right) / T$ is a finite dimensional extension of $T$. As in the proof of Corollary 6.18, the CM property therefore forces $U_{n} \subseteq T$. Hence $U \subseteq T$, as claimed.

It remains to get a detailed understanding of the graded pieces $U_{n}$ of $U$. To this end, define a graded subspace $W$ of $T$ by

$$
W_{n}:= \begin{cases}g^{h(n)} T_{n-h(n)} & \text { if } U_{n} \neq 0 \\ 0 & \text { otherwise. }\end{cases}
$$

Let $n, m$ be such that $W_{n}, W_{m} \neq 0$. Using the equation $h(n+m) \leqslant h(n)+h(m)$ we have

$$
W_{n} W_{m}=g^{h(n)+h(m)} T_{n-h(n)} T_{m-h(m)} \subseteq g^{h(n+m)} T_{m+n-h(n+m)}=W_{n+m} .
$$


Thus $W$ is a ring. The definition of $h$ and $g$-divisibility of $T$ force $U \subseteq W$ and we may clearly extend $\theta$ to an isomorphism $\theta: W^{(K)} \cong T^{(M)}$.

We next want to understand the structure of $W$ over its Veronese subalgebras. By [AS, Lemma 4.10], $U$ is a finitely generated left and right module over $U^{(K)}$. For $1 \leqslant i \leqslant K-1$, let $U(i)=\oplus_{n} U_{n K+i}$. This is finitely generated as a right $U^{(K)}$-module, say by $\bigoplus_{n=0}^{n_{i}} U_{n K+i}$. Using (7.3), it follows for $n \geqslant n_{i}$ that

$$
\begin{aligned}
h(n K+i) & =\min _{n^{\prime} \leqslant n_{i}}\left(h\left(n^{\prime} K+i\right)+h\left(\left(n-n^{\prime}\right) K\right)\right) \\
& =\left(n-n_{i}\right) h(K)+\min _{n^{\prime} \leqslant n_{i}}\left(h\left(n^{\prime} K+i\right)+h\left(\left(n_{i}-n^{\prime}\right) K\right)\right)=\left(n-n_{i}\right) h(K)+h\left(n_{i} K+i\right) .
\end{aligned}
$$

Consequently, $W_{n K+i}=W_{n_{i} K+i} W_{\left(n-n_{i}\right) K}=W_{\left(n-n_{i}\right) K} W_{n_{i} K+i}$ for $1 \leqslant i \leqslant K-1$ and $n \geqslant n_{i}$, and so $W$ is a finitely generated left and right $W^{(K)}$-module. Now $\theta\left(W^{(K)}\right)=T^{(M)}$ is a finitely generated module over $V=\theta\left(U^{(K)}\right)$ on both sides, and so $W^{(K)}$ is a finitely generated $U^{(K)}$-module on both sides. Thus $W$ is finitely generated as a $U^{(K)}$-module and hence as a $U$-module on both sides. Since $T \subseteq R$, it is clear that $Q_{\mathrm{gr}}(U)=Q_{\mathrm{gr}}(W)=\left(Q_{\mathrm{gr}}(T)\right)^{(j+1)}$. 1 $\mathrm{A}$ As $R$ and therefore $U$ are maximal orders, $U=W$. It follows that $V=\theta\left(W^{(K)}\right)=T^{(M)}$.

Finally, for $n \in \mathbb{N}$, we have

$$
R_{n}=\phi_{j}^{-1}\left(W_{(j+1) n}\right)=g^{h((j+1) n)-n j} T_{(j+1) n-h((j+1) n)} .
$$

Pick $m \in \mathbb{N}$. Then, as $(j+1) N=K$ and $h(K m)=h(K) m$, we have

$$
R_{N m}=g^{h(K m)-N j m} T_{K m-h(K m)}=g^{(N-M) m} T_{M m}
$$

and so $R^{(N)}=\mathbb{k}\left\langle g^{N-M} T_{M}\right\rangle \cong T^{(M)}$. As $R_{N} \supseteq T_{N}$ we have $M \geqslant N$ and the result is proved.

(2) Our notation in this part of the proof is that Veronese rings are not regraded; so $\left(A^{(K)}\right)_{K n}=A_{K n}$ for all $n \in \mathbb{N}$ and $g \in T_{d}=S_{d}$. A number of the steps of the proof exactly parallel those from Part (1), in which case the proof is left to the reader.

For $j \in \mathbb{N}$, extend $\phi_{j}$ to a map $\psi_{j}: Q_{\mathrm{gr}}(S) \rightarrow Q_{\mathrm{gr}}\left(S^{(j d+1)}\right)$ by $\psi_{j}(f)=f g^{j n}$ for all $f \in Q_{\mathrm{gr}}(S)_{n}$. As in Part (1), each $\psi_{j}$ is an injective graded algebra homomorphism, and $\psi_{j}(A) \subset S_{(g)}$ for some $j$. Set $X=\psi_{j}(A)$. Define $h: \mathbb{N} \rightarrow \mathbb{N} \cup\{\infty\}$ by

$$
h(n):=\max \left\{i: X_{n} \subseteq g^{i} S_{(g)}\right\}, \text { with } h(n)=\infty \text { if } X_{n}=0 .
$$

As in Part (1), $h(n+m) \leqslant h(n)+h(m)$ for all $n, m \in \mathbb{N}$ and $h(n)$ satisfies the analogue of (7.3).

Set $R:=A^{(d)} \supseteq T:=S^{(d)}$. Then $R$ and $U:=\psi_{j}\left(A^{(d)}\right) \subset T_{(g)}$ have the same properties as their counterparts in Part (1) of the proof. In particular, there is an integer $K$, which we may take to be a multiple of $d$, so that $h(n K)=n h(K)$ for all $n \in \mathbb{N}$. Further, there is a graded ring homomorphism $\theta: U^{(K)} \rightarrow T_{(g)}$ with $\theta(x)=g^{-h(\operatorname{deg} x)} x$ for all homogeneous $x \in U^{(K)}$ and, moreover, $V=\theta\left(U^{(K)}\right) \nsubseteq \mathbb{k}+g T_{(g)}$. Set

\footnotetext{
${ }^{1}$ As an aside for later use, we remark that so far we have used only that $R$ is cg noetherian and that $T$ is a minimal elliptic surface.
} 
$Z=\widehat{V[g]}$. Using the fact that $T$ is a minimal elliptic surface by Theorem 6.16, the arguments of Part (1) show that $Z=T$ is a finitely generated $V$-module on both sides. Moreover $U \subseteq T$.

We next claim that $X \subseteq S$. Certainly there exists $L$ such that $X^{(L)}=U^{(L)} \subseteq T^{(L)}$ (in fact $L=(d j+1) d$ will work). As $A \supseteq S$, if $X_{n} \neq 0$ then $X_{n} \supseteq g^{a} S_{b}$, for some $a, b \geqslant 1$. Now the proof from Part (1) that $U \subseteq T$ can be used essentially unchanged to show that $X \subseteq S$.

Now define $Y=\oplus_{n \geqslant 0} Y_{n}$ where

$$
Y_{n}:= \begin{cases}g^{h(n)} S_{n-d h(n)} & \text { if } X_{n} \neq 0 \\ 0 & \text { else. }\end{cases}
$$

Clearly $Y_{n} \supseteq X_{n}$ for all $n$ by $g$-divisibility of $S$. Using the inequality $h(n+m) \leqslant h(n)+h(m)$ gives

$$
Y_{n} Y_{m}=g^{h(n)+h(m)} S_{n+m-d(h(n)+h(m))} \subseteq g^{h(n+m)} S_{n+m-d h(n+m)}=Y_{n+m},
$$

and so $Y$ is a $\mathbb{k}$-algebra.

Recall that $T=Z$ is a finitely generated module over $V=\theta\left(U^{(K)}\right)$. As in Part (1), each $U(i)=\oplus_{n} U_{n K+i}$ is finitely generated as a right $U^{(K)}$-module, say by $\oplus_{n=0}^{n_{i}} U_{n K+i}$. Equation (7.5) then follows formally and this now ensures that $Y$ is a finitely generated $Y^{(K)}$-module on both sides. It follows that $Y^{(K)}$ is a finitely generated $U^{(K)}$-module on both sides and thus $Y$ is a finitely generated $X$-module on both sides. As in Part (1), $Q_{\mathrm{gr}}(Y)=Q_{\mathrm{gr}}(X)$ and as $X \cong A$ is a maximal order, $Y=X$. This proves the first assertion of Part (2). The final sentence follows as in Part (1).

One of the significant consequences of [RSS2] is that graded maximal orders contained in $T$ are automatically noetherian. We conjecture that in the main theorems of this paper, Theorems [5.5 and 7.1 as well as Corollary 6.18, the same is true for overrings. More precisely:

Conjecture 7.6. Theorem 7.1(1) holds even if $R$, respectively $A$, is not assumed to be noetherian. In particular, and in the notation of the theorem, finitely graded maximal orders $T \subseteq R \subset Q_{\mathrm{gr}}(T)$ are automatically noetherian. Similar comments hold for overrings of $S$.

We next give a couple of examples that show that one cannot easily improve on Theorem [7.1. As usual, given a subset $V$ in a $k$-algebra $A$ we write $k\langle V\rangle$ for the $k$-algebra generated by $V$. Following the discussion at the beginning of the section, and by analogy with [RSS2, Proposition 9.1], one might hope that any $\mathrm{cg}$ noetherian overring $T \subseteq U \subset Q_{\mathrm{gr}}(T)$ would have the form $U=\mathbb{k}\left\langle T_{n+1} g^{-n}\right\rangle$ for some $n$. As the next example shows, this is not the case.

Example 7.7. Let $T:=S k l^{(3)}$, the 3 -Veronese of a quadratic Sklyanin algebra and set

$$
U:=\mathbb{k}\left\langle T_{1}, g^{-1} T_{3}\right\rangle=\mathbb{k}+T_{1}+T_{3} g^{-1}+T_{4} g^{-1}+T_{6} g^{-2}+T_{7} g^{-2}+\ldots
$$

Then $U$ is a noetherian cg maximal order with $U \supsetneq T$ that cannot be written as $U=\mathbb{k}\left\langle T_{n+1} g^{-n}\right\rangle$ for any $n$. However, up to a change of grading, $U^{(2)} \cong T^{(3)}$. 
Proof. Note that $\phi_{1}(U)=R=\mathbb{k}\left\langle g T_{1}, g T_{3}\right\rangle$, in the notation from the proof of Theorem 7.1. Since $g$ is central and $T$ is generated in degree one it is easy to see that $\left(g T_{1}\right)^{2}=g^{2} T_{2} \subseteq g T_{3}$ and hence that $R^{(4)}=\mathbb{k}\left\langle g T_{3}\right\rangle \cong T^{(3)}$. Since $T^{(3)}$ is noetherian, so are $R^{(4)}$ and hence $R$ by [AS, Lemma 4.10(3)]. It is an easy exercise to see that $U$ is not generated by any set $T_{n+1} g^{-n}$.

It remains to prove that $R$ is a maximal order. Throughout the proof we keep the grading from $T$; thus $R$ is generated in degrees 2 and 4 , while $\operatorname{deg}(g)=1$. First, by [BH, Proposition 3.37], $R^{(4)} \cong T^{(3)}$ is a maximal order. So, suppose that $R \subseteq A$ is an equivalent order. Then $A^{(4)}$ is equivalent to $R^{(4)}$ by [BH, Lemma 3.32] and hence $A^{(4)}=R^{(4)}$. We next show that $A \subseteq T$. Let $a \in A \backslash T$ be homogeneous. Since $A^{(4)}=R^{(4)}$, clearly $\operatorname{deg}(a)=4 e+2$ for some $e \in \mathbb{N}$. Since $g^{2} \in R$ we have $g^{2} a \in R^{(4)} \subseteq \mathbb{k}+g T$ and so $a \in g^{-1} T$. As $a \notin T$, then $a=g^{-1} x$ for some $x \in T \backslash g T$. Notice that $g$ does not divide $x^{2}$ since $g T$ is a completely prime ideal. Hence $a^{2}=g^{-2} x^{2} \notin T$; a contradiction. Thus $A \subseteq T$.

Now let $a \in A_{4 e+2}$ for some $e \in \mathbb{N}$. Write $a=g^{u} v$ with $u$ as large as possible. Writing $a$ as a sum of terms we may also assume that $v=v_{1} v_{2}$ with $v_{1}, v_{2} \in T$ and $\operatorname{deg} v_{1}=1$. Then $g^{2 u} v^{2} \in A_{8 e+4}=R_{8 e+4}=g^{2 e+1} T_{6 e+3}$. As $g T$ is completely prime, $g$ does not divide $v^{2}$ and so $2 u \geqslant 2 e+1$, hence $u>e$. Thus we can rewrite $a=g^{e+1} v_{1} w_{2}=\left(g v_{1}\right)\left(g^{e} w_{2}\right)$ where, now, $w_{2} \in T_{3 e}$. Thus, $g^{e} w_{2} \in g^{e} T_{3 e}=R_{4 e}$ and $a \in R^{(4)}\left\langle g T_{1}\right\rangle=R$, as required.

As the next example shows, if one merely assumes that $U$ is a noetherian cg overring of $T$ in Theorem 7.1 then more complicated examples can arise.

Example 7.8. Let $T:=S k l^{(3)}$, the 3 -Veronese of a quadratic Sklyanin algebra with factor $B:=T / g T$. Set $R:=(g T)^{(2)}+T^{(4)}$ and let $U:=\phi_{1}^{-1}(R)$, where $\phi_{1}$ is defined by (7.2). Then $U$ and $R$ are noetherian cg rings such that $\widehat{R}^{(2)}=T^{(2)}$ is a finitely generated $R$-module on both sides. Similarly, $T \subseteq U \subseteq V:=\phi_{1}^{-1}\left(T^{(2)}\right)$, with $V$ a finitely generated $U$-module on both sides. However, both $\widehat{R}^{(2)} / R$ and $V / U$ are infinite dimensional, so $U$ has noetherian overrings that are substantially larger than itself.

Proof. Clearly $\phi_{1}(T)=\mathbb{k}\left\langle g T_{1}\right\rangle \subseteq \mathbb{k}+(g T)^{(2)} \subseteq R \subseteq T^{(2)}=\phi_{1}(V)$. Thus $T \subseteq U \subseteq V$ and all the assertions about $U$ follow from the corresponding assertions about $R$.

Since $g^{2} T^{(2)} \subset R \subseteq T^{(2)}=\widehat{T}^{(2)}$, certainly $\widehat{R}^{(2)}=T^{(2)}$. On the other hand as $T^{(4)} \subset R \subseteq T^{(2)}$ and $T^{(2)}$ is a (left and right) noetherian $T^{(4)}$-module, so is $R$. Hence $R$ is a noetherian ring and $\widehat{R}$ is a noetherian $R$-module on both sides. It remains to prove that $\operatorname{dim}_{\mathbb{k}} T^{(2)} / R=\infty$. Since $(g T)^{(2)}$ is an ideal of $R$, it suffices to prove that $T^{(2)} /(g T)^{(2)} \cong B^{(2)}$ is not a finite dimensional extension of $R /(g T)^{(2)}$. Since

$$
R /(g T)^{(2)}=\frac{(g T)^{(2)}+T^{(4)}}{(g T)^{(2)}} \cong \frac{T^{(4)}}{(g T)^{(2)} \cap T^{(4)}}=\frac{T^{(4)}}{(g T)^{(4)}}=B^{(4)}
$$

the assertion follows.

Remark 7.9. Let $S:=S k l$ be a quadratic Sklyanin algebra, and let $T:=S^{(3)}$. For the purposes of this remark, define a minimal model to be a cg noetherian algebra $T^{\prime}$ containing $g$ and birational to $T$ with the 
property that if $R^{\prime}$ is cg noetherian with $T^{\prime} \varsubsetneqq R^{\prime} \subset T_{(g)}^{\prime}$, then $T^{\prime}=R^{\prime}$ (thus we are not assuming that $T^{\prime}$ is elliptic). The ultimate aim in the present project is, of course, to classify all the minimal models birational to $T$ (or $S$ ) and then to prove that any finitely graded maximal order $R$ birational to $S$ can be obtained from such a minimal model by blowing up (including virtual blowing up as in RSS2]) finitely many points on the elliptic curve $E$.

However, unlike the commutative situation, we expect there to be more minimal models than just the noncommutative projective plane and quadrics. More precisely, reflexive right ideals $P$ of $S$ have been classified through formal moduli spaces [NS], with a discrete invariant $c(P)$ analogous to a second Chern class and a continuous one deforming a Hilbert scheme of points. By analogy with work on the Weyl algebra (see for example [BW]), we hope that

$$
\operatorname{End}_{S}(P) \cong \operatorname{End}_{S}\left(P^{\prime}\right) \Longleftrightarrow c(P)=c\left(P^{\prime}\right) \quad \text { for reflexive right ideals } P, P^{\prime}
$$

Analogous results should hold if $S$ is replaced by $T$ or $Q_{V d B}$ or their Veronese rings.

The expectation is that the corresponding endomorphism rings $\operatorname{End}(P)$ will then give all minimal models birational to $S$. For elliptic algebras a stronger conjecture will be given in Conjecture 8.13 .

\section{General overrings in the locally hereditary Case}

The arguments of Section 4 can also be used to obtain information on the structure of arbitrary cg noetherian overrings of non-minimal elliptic surfaces $T$ provided that $T$ is locally hereditary in the sense that $\operatorname{gldim} T^{\circ}=1$. The main result of this section is an analogue of Theorem 1.2, that is, the classical result that any birational morphism of smooth projective surfaces is a composition of finitely many monoidal transformations.

We recall that for elliptic algebras $T$ there is a good analogue of Castelnuovo's theorem on contracting rational curves of self-intersection $(-1)$. In order to state this we note that, if $T$ is an elliptic algebra such that qgr- $T$ is smooth in the sense of Definition [6.5, then there is a well-defined intersection product [MS], on qgr- $T$. This is given by $(M \cdot N):=\sum_{i=0}^{\infty}(-1)^{i+1} \operatorname{dim} \operatorname{Ext}_{\text {qgr- } T}^{i}(M, N)$, for $M, N \in \operatorname{gr}-T$. The noncommutative version of Castelnuovo's theorem is as follows.

Theorem 8.1. ([RSS4, Theorems 1.4, 1.5, and 8.1, Lemma 8.2]) Let $T$ be an elliptic algebra so that qgr- $T$ is smooth, and let $L$ be a line module so that $L$ has self-intersection $(L \cdot L)=-1$. Then there is an elliptic algebra $\tilde{T}$ with $T \subset \widetilde{T} \subset T_{(g)}$ and so that $\tilde{T} / T \cong \oplus_{i \geqslant 1} L[-i]$ as right $T$-modules. Further, $\widetilde{T}$ is the maximal submodule of $Q_{g r}(T)$ so that $\widetilde{T} / T$ is isomorphic to a direct sum of shifts of $L$, and qgr- $\widetilde{T}$ is smooth.

We refer to the construction of $\widetilde{T}$ from $T$ given in Theorem 8.1 as blowing down or contracting the line $L$. We now state the main result of this section. 
Theorem 8.2. Let $T$ be an elliptic algebra of degree $\geqslant 3$ such that $T^{\circ}$ is hereditary and let $T \subseteq R \subset T_{(g)}$ be any $g$-divisible finitely graded overring. Then $R$ is obtained from $T$ by successively blowing down finitely many line modules $L$ of self-intersection $(L \cdot L)=-1$. In particular, $R$ is elliptic.

We immediately note a simple corollary obtained by combining the theorem with Lemma 3.9,

Corollary 8.3. Let $T$ be an elliptic algebra of degree $\geqslant 3$ such that $T^{\circ}$ is hereditary and let $T \subseteq R \subset T_{(g)}$ be any noetherian cg overring. Then there is an extension $R \subseteq R^{\prime} \subset T_{(g)}$, finitely generated as a left and right $R$-module, such that $R^{\prime}$ is obtained from $T$ by successively blowing down finitely many line modules $L$ of self-intersection $(-1)$.

The proof of Theorem 8.2 will take the whole section and we assume throughout that the hypotheses of the theorem are satisfied. To begin, we may assume that $T \neq R$ and, by Proposition 3.2 pick a critical module $L$ so that some shift of $L$ is contained in $R / T$ and so that $\mathcal{S}:=L^{\circ}$ is a simple submodule of $R^{\circ} / T^{\circ}$. Without loss of generality, we may shift $L$ so that $\min \left\{n: L_{n} \neq 0\right\}=0$. If no such $L$ is a line module (by Proposition 3.2 this is equivalent to saying that $L$ has multiplicity $d(L)>1$ ), then the conditions of Hypothesis 4.1 are automatically satisfied. In this case Proposition 4.8 applies and leads to a contradiction. Thus, we can and will assume that $L$ is a line module.

Remark 8.4. We note that by [RSS4, Lemmas 5.2 and 5.4], $L$ is CM with $j(M)=1$.

The heart of the proof will be to prove the following fact.

Proposition 8.5. In the situation above, $(L \cdot L)=-1$.

Before proving the proposition, we will show that this quickly implies the theorem.

Proof of Theorem 8.2. Let $T \subset M \subseteq R$ so that $M / T \cong L[-i]$ for some $i$.

Applying Theorem 8.1 we can blow down $T$ at $L$ to obtain a second elliptic algebra $U:=\widetilde{T} \supsetneqq T$ such that $U / T \cong \oplus_{n \geqslant 1} L[-n]$. Therefore $U^{\circ} / T^{\circ} \cong \oplus_{n \geqslant 1} \mathcal{S}_{n}$ with $\mathcal{S}_{n} \cong L^{\circ}$ for all $n$. However, by [Go, Theorem 5] every overring of $T^{\circ}$ is obtained by a torsion-theoretic localisation at some set $X$ of simple modules. In particular, the overring of $T^{\circ}$ generated by $M^{\circ}$ is such an overring and hence must equal $U^{\circ}$. Consequently, $U^{\circ} \subseteq R^{\circ}$. Since $U$ is $g$-divisible, $U=\Phi\left(U^{\circ}\right) \subseteq \Phi\left(R^{\circ}\right)=R$.

By [Ku, Proposition 1.6], $U^{\circ}$ is hereditary. Since $\infty>\operatorname{dim} R_{1} \geqslant \operatorname{dim} U_{1}>\operatorname{dim} T_{1}$, we may now induct on $\operatorname{dim} U_{1}$ to conclude that $R$ is obtained from $U$ (and hence $T$ ) by blowing down a finite number of line modules of self-intersection $(-1)$. This completes the proof of the theorem.

It remains to prove Proposition 8.5 , for which we need several lemmas. Note that, by [RSS4, Corollary 6.6 and Lemma 5.5],

$$
(L \cdot L)=-1 \Longleftrightarrow \operatorname{Ext}_{T}^{1}(L, L)=0 .
$$


So the proof of Proposition 8.5 amounts to describing this Ext group. We note that Goodearl's result also applies to the rings $T^{\circ} \subseteq R^{\circ}$ and implies, in particular, the following fact: Suppose that $T^{\circ} \subseteq N^{\circ} \subseteq \operatorname{Fr}\left(T^{\circ}\right)$ is a finitely generated module extension such that $N^{\circ} / T^{\circ}$ has a composition series with factors consisting entirely of copies of $\mathcal{S}=L^{\circ}$. Then $N \subseteq R^{\circ}$.

Applying $\Phi$ this gives:

Lemma 8.7. Suppose that $P$ is a $T$-module with $T \subseteq P \subseteq T_{(g)}$ and assume that $P / T$ has a finite composition series with all factors isomorphic to shifts $L[r]$ of $L$. Then $P \subseteq R$.

We note also that the possibilities for $\operatorname{Ext}_{T}^{1}(L, L)$ are quite limited.

Lemma 8.8. If $T$ is any elliptic algebra and $L$ is any right $T$-line module, then as a $g$-module $\operatorname{Ext}_{T}^{1}(L, L)$ is isomorphic to one of:

$$
0, \quad \mathbb{k}[g], \quad g^{-1} \mathbb{k}[g], \quad g^{-1} \mathbb{k}[g] \oplus \mathbb{k}[g] .
$$

In particular, $\operatorname{Ext}_{T}^{1}(L, L)$ is g-torsionfree.

Proof. Let $\bar{L}=L / L g$, and note that, by [RSS4, Lemma 4.7], there is an exact sequence

$$
0 \longrightarrow \operatorname{Ext}_{T}^{1}(L, L[-1]) \stackrel{\cdot g}{\longrightarrow} \operatorname{Ext}_{T}^{1}(L, L) \stackrel{\delta}{\longrightarrow} \operatorname{Ext}_{T / g T}^{1}(\bar{L}, \bar{L}) .
$$

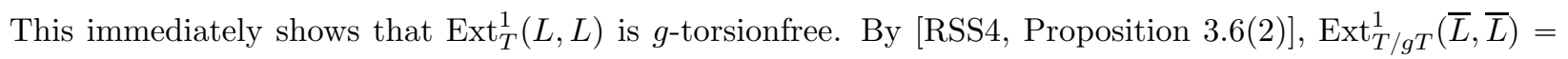
$\mathbb{k} \oplus \mathbb{k}[1]$. Thus, $\operatorname{Im} \delta$ is a graded subspace of $\mathbb{k} \oplus \mathbb{k}[1]$. Since $\operatorname{Ext}_{T}^{1}(L, L)$ is left bounded and $g$-torsionfree, this gives the four claimed possibilities.

Lemma 8.9. $\operatorname{Ext}_{T}^{1}(L, L)_{-1}=0$.

Proof. Suppose the contrary: so $\operatorname{Ext}_{T}^{1}(L, L[-1])_{0}=\operatorname{Ext}_{T}^{1}(L, L)_{-1} \neq 0$ and there is a nonsplit extension

$$
0 \rightarrow L[-1] \rightarrow M \rightarrow L \rightarrow 0
$$

Since $L$ is CM by Remark 8.4, this induces an exact sequence

$$
0 \rightarrow \operatorname{Ext}_{T}^{1}(L, T) \rightarrow \operatorname{Ext}_{T}^{1}(M, T) \rightarrow \operatorname{Ext}_{T}^{1}(L[-1], T) \rightarrow 0 .
$$

By Lemma $2.5 \operatorname{Ext}_{T}^{1}(L, T)_{1}=\mathbb{k}$, say given by the nonsplit extension $[Y]: 0 \rightarrow T \rightarrow Y \rightarrow L[-1] \rightarrow 0$. By the displayed equation this lifts to a (necessarily nonsplit) extension, say $0 \rightarrow T \rightarrow X \rightarrow M \rightarrow 0$.

We claim that $Y$ is (Goldie) torsionfree. Indeed, if $Y$ has a nonzero torsion submodule $N$, then $N$ would be canonically isomorphic to a submodule of $L[-1]$ and hence $\operatorname{Ext}_{T}^{1}(L[-1] / N, T) \neq 0$. As $L[-1]$ is 2-critical, $\operatorname{GKdim}(L[-1] / N)<2$ and so this contradicts the fact that $T$ is CM [RSS3, Proposition 2.4]. This proves the claim; in particular $Y^{\circ}$ is also torsionfree.

By Lemma 8.8, $\operatorname{Ext}_{T}^{1}(L, L)$ is $g$-torsionfree and so the extension $0 \rightarrow \mathcal{S} \rightarrow M^{\circ} \rightarrow \mathcal{S} \rightarrow 0$ is nonsplit. Thus by Lemma 4.5. $X^{\circ}$ is (Goldie) torsionfree. As $L$ is $g$-torsionfree, so are $M$ and $X$. Thus, if $X$ had nonzero 
Goldie torsion, then so would $X\left[g^{-1}\right]$ and $X^{\circ}$, giving a contradiction. Therefore, $X$ must also be torsionfree. Finally, by Lemma 8.7, this implies that $X \subseteq R$. Since $\operatorname{dim} X_{0}=2$ but $R_{0}=\mathbb{k}$, this is a contradiction.

Combining the last two lemmas gives:

Corollary 8.10. Either $\operatorname{Ext}_{T}^{1}(L, L)=0$ or $\operatorname{Ext}_{T}^{1}(L, L)=\mathbb{k}[g]$.

We now come to the key step in the proof of Proposition 8.5.

Proposition 8.11. In Corollary [8.10, suppose that $\operatorname{Ext}_{T}^{1}(L, L) \neq 0$. Then for all $n \in \mathbb{Z}_{\geqslant 1}$, there is a graded CM T-module $L(n)$, where:

(1) $L(1)=L$;

(2) there is a nonsplit extension $0 \rightarrow L(n) \rightarrow L(n+1) \rightarrow L \rightarrow 0$;

(3) $L(n)^{\circ}$ is essential in $L(n+1)^{\circ}$.

Proof. We will show by induction that $L(n)$ exists with the claimed properties and that $\operatorname{Ext}_{T}^{1}(L, L(n)) \cong$ $\operatorname{Hom}_{T}(L, L(n)) \cong \mathbb{k}[g]$. The base case $n=1$ holds by Remark 8.4 and Corollary 8.10,

Suppose that $N=L(n)$ has been constructed with the given properties. Since $\operatorname{Ext}_{T}^{1}(L, N)=\mathbb{k}[g]$, there is a nonsplit exact sequence $0 \rightarrow N \rightarrow M \rightarrow L \rightarrow 0$ and so certainly $M=L(n+1)$ exists, and is CM by induction. By Lemma $8.8 \operatorname{Ext}_{T}^{1}(L, N)$ is $g$-torsionfree, so $M^{\circ} ¥ N^{\circ} \oplus \mathcal{S}$. As $L^{\circ}=\mathcal{S}$ is simple, $N^{\circ}$ is essential in $M^{\circ}$.

Consider the localised exact sequence

$$
\begin{aligned}
0 \longrightarrow \operatorname{Hom}_{T^{\circ}}\left(\mathcal{S}, N^{\circ}\right) \longrightarrow \operatorname{Hom}_{T^{\circ}}\left(\mathcal{S}, M^{\circ}\right) \stackrel{\alpha}{\longrightarrow} \operatorname{Hom}_{T^{\circ}}(\mathcal{S}, \mathcal{S}) \stackrel{\beta}{\longrightarrow} \\
\longrightarrow \operatorname{Ext}_{T^{\circ}}^{1}\left(\mathcal{S}, N^{\circ}\right) \longrightarrow \operatorname{Ext}_{T^{\circ}}^{1}\left(\mathcal{S}, M^{\circ}\right) \longrightarrow \operatorname{Ext}_{T^{\circ}}^{1}(\mathcal{S}, \mathcal{S}) \longrightarrow 0
\end{aligned}
$$

As noted above, $M^{\circ}$ is a nonsplit extension, and so

$$
\operatorname{Hom}_{T^{\circ}}\left(\mathcal{S}, M^{\circ}\right) \cong \operatorname{Hom}_{T^{\circ}}\left(\mathcal{S}, N^{\circ}\right)=\mathbb{k} .
$$

Thus, $\alpha=0$. Thus $\beta$ is injective, and since $\operatorname{Ext}_{T^{\circ}}^{1}\left(\mathcal{S}, N^{\circ}\right)=\mathbb{k}$ by induction, $\beta$ is even an isomorphism. It follows that $\operatorname{Ext}_{T^{\circ}}^{1}\left(\mathcal{S}, M^{\circ}\right) \cong \operatorname{Ext}_{T^{\circ}}^{1}(\mathcal{S}, \mathcal{S})=\mathbb{k}$.

Consider now the long exact sequence

$$
\begin{aligned}
0 \longrightarrow & \operatorname{Hom}_{T}(L, N) \longrightarrow \operatorname{Hom}_{T}(L, M) \stackrel{\hat{\alpha}}{\longrightarrow} \operatorname{Hom}_{T}(L, L) \stackrel{\hat{\beta}}{\longrightarrow} \\
& \longrightarrow \operatorname{Ext}_{T}^{1}(L, N) \longrightarrow \operatorname{Ext}_{T}^{1}(L, M) \longrightarrow \operatorname{Ext}_{T}^{1}(L, L)
\end{aligned}
$$

Since $\alpha=0$, the image of $\widehat{\alpha}$ must be a $g$-torsion submodule of $\operatorname{Ext}_{T}^{1}(L, L) \cong \mathbb{k}[g]$, and so $\widehat{\alpha}=0$. Thus $\operatorname{Hom}_{T}(L, M) \cong \operatorname{Hom}_{T}(L, N) \cong \mathbb{k}[g]$ by induction, again. Further, $\widehat{\beta}$ is an injective map between the graded spaces $\operatorname{Hom}_{T}(L, L) \cong \mathbb{k}[g]$ and $\operatorname{Ext}_{T}^{1}(L, N) \cong \mathbb{k}[g]$ and so is an isomorphism. Thus $\operatorname{Ext}_{T}^{1}(L, M) \hookrightarrow$ $\operatorname{Ext}_{T}^{1}(L, L) \cong \mathbb{k}[g]$ and so $\operatorname{Ext}_{T}^{1}(L, M) \cong g^{m} \mathbb{k}[g]$ for some $m \geqslant 0$. 
Finally, write $\bar{L}:=L / L g$ and consider the exact sequence

$$
\operatorname{Ext}_{T}^{1}(L, M)[-1] \stackrel{\cdot g}{\longrightarrow} \operatorname{Ext}_{T}^{1}(L, M) \stackrel{\gamma}{\longrightarrow} \operatorname{Ext}_{T / g T}^{1}(\bar{L}, \bar{M})
$$

given by [RSS4, Lemma 4.7]. Since $\bar{M}$ has a filtration whose factors are $n+1$ copies of the point module $\bar{L}$, [RSS4, Proposition 3.6(2)] implies that $\operatorname{Im} \gamma \subseteq(\mathbb{k}[1] \oplus \mathbb{k})^{(n+1)}$. Thus, since $\operatorname{Ext}_{T}^{1}(L, M)$ is left bounded and $g$-torsionfree the map $\cdot g$ is an injection and the graded version of Nakayama's Lemma implies that generators of $\operatorname{Im}(\gamma)$ pull back to generators of $\operatorname{Ext}_{T}^{1}(L, M)$. Hence $m=0$, completing the proof of the induction step.

Proof of Proposition 8.5, Suppose that $\operatorname{Ext}_{T}^{1}(L, L) \neq 0$. Then modules $L(n)$ as in Proposition 8.11 exist for all $n$. We claim that there is a torsionfree extension $0 \rightarrow T \rightarrow X(n) \rightarrow L(n)[-1] \rightarrow 0$ for all $n$.

As in the proof of Lemma 8.9, certainly $X(1)$ exists. By induction, assume that $X(n)$ exists and let $\xi_{n}$ be the corresponding element of $\operatorname{Ext}_{T}^{1}(L(n), T)$. As $L(n)$ is CM we have the exact sequence

$$
0 \rightarrow \operatorname{Ext}_{T}^{1}(L, T) \rightarrow \operatorname{Ext}_{T}^{1}(L(n+1), T) \rightarrow \operatorname{Ext}_{T}^{1}(L(n), T) \rightarrow 0 .
$$

Let $\xi_{n+1}$ be a preimage of $\xi_{n}$ in $\operatorname{Ext}_{T}^{1}(L(n+1), T)$ and let $0 \rightarrow T \rightarrow X(n+1) \rightarrow L(n+1) \rightarrow 0$ be the corresponding extension. Under localisation this gives the extension $0 \rightarrow T^{\circ} \rightarrow X(n+1)^{\circ} \rightarrow L(n+1)^{\circ} \rightarrow 0$. By Lemma 4.5 and Proposition $8.11(3), X(n+1)^{\circ}$ is torsionfree, and as in the final paragraph of the proof of Lemma $8.9 X(n+1)$ must be torsionfree, as claimed.

Thus $X(n) \subseteq R$ for all $n$, by Lemma 8.7. But $\operatorname{dim} X(n)_{1} \geqslant n$, whence $\operatorname{dim} R_{1}=\infty$. This gives the required contradiction and completes the proof of the proposition and hence that of Theorem 8.2 .

We also note that Theorem 8.2 provides the following variant of Theorem 5.5 for elliptic algebras of degree at least 3 .

Theorem 8.12. Let $T$ be an elliptic algebra of degree $\geqslant 3$ such that $T^{\circ}$ is hereditary and $T$ has no line modules of self-intersection $(-1)$. Then $T$ is a minimal elliptic surface.

Proof. Suppose there exists a cg noetherian ring $R$ with $T \subsetneq R \subset T_{(g)}$. By Lemma 3.9 we can replace $R$ by $\hat{R}$ and assume that $R$ is also $g$-divisible. Now Theorem 8.2 implies that $R=T$.

We end by making the following conjecture. This may be compared with Remark 7.9 where the algebras are not assumed to be elliptic.

Conjecture 8.13. If $T, T^{\prime}$ are two elliptic algebras with $Q_{g r}(T)=Q_{g r}\left(T^{\prime}\right)$, then they are related, up to isomorphism, by a finite series of blowdowns and blowups. 


\section{GelFAnd-Kirillov Dimension}

In this final section, we consider the Gelfand-Kirillov (GK) dimension of overrings of elliptic algebras. See KL for the basic theory of GK-dimension. Let $T$ be a minimal elliptic surface; by definition GKdim $T=3$. Theorem 6.16 and Corollary 6.18 can be viewed as saying that any noetherian graded algebra lying strictly between $T$ and $Q_{g r}(T)$ must be significantly larger than $T$. In this section we prove that this is true in the sense of GK-dimension as well: any proper noetherian graded overring of $T$ contained in $T_{(g)}$ has GKdimension $\geqslant 4$. We also show that, when $T=S k l^{(3)}$ or $T=S k l^{(4)}$ or $T=Q_{V d B}^{(2)}$, any proper overring of $T^{\circ}$ has GK-dimension $\geqslant 3$.

We first give some elementary computations on linear systems on elliptic curves. Let $E$ be an elliptic curve and let $K=\mathbb{k}(E)$. If $f \in K$, we write

$$
(f)=(f)_{0}-(f)_{\infty}
$$

for the divisor of $f$, where both $(f)_{0}$ and $(f)_{\infty}$ are effective and of minimal degree. If $D$ is a divisor on $E$, we write $|D|=H^{0}(E, \mathcal{O}(D))$, which we identify with $\{f \in K:(f)+D \geqslant 0\}$.

If $y \in K$ then

$$
y|D|=\left\{f \in K: y^{-1} f \in|D|\right\}=\{f:(f)+D \geqslant(y)\}=|D-(y)| \subseteq\left|D+(y)_{\infty}\right|
$$

We need the following elementary lemmas.

Lemma 9.3. Let $D, D^{\prime}$ be divisors on $E$ such that $\operatorname{deg} \inf \left\{D, D^{\prime}\right\}>0$. Then $|D|+\left|D^{\prime}\right|=\left|\sup \left\{D, D^{\prime}\right\}\right|$.

Proof. Certainly $|D|,\left|D^{\prime}\right| \subseteq\left|\sup \left\{D, D^{\prime}\right\}\right|$. For the other inclusion, we count dimensions. We have:

$$
|D| \cap\left|D^{\prime}\right|=\left\{f \in K:(f) \geqslant-D,-D^{\prime}\right\}=\left\{f:(f) \geqslant \sup \left\{-D,-D^{\prime}\right\}\right\}=\left|\inf \left\{D, D^{\prime}\right\}\right|
$$

So

$$
\begin{aligned}
\operatorname{dim}\left(|D|+\left|D^{\prime}\right|\right) & =\operatorname{dim}|D|+\operatorname{dim}\left|D^{\prime}\right|-\operatorname{dim}\left(|D| \cap\left|D^{\prime}\right|\right) \\
& =\operatorname{deg} D+\operatorname{deg} D^{\prime}-\operatorname{deg} \inf \left\{D, D^{\prime}\right\} \quad \text { by Riemann-Roch and hypothesis on } \inf \left\{D, D^{\prime}\right\} \\
& =\operatorname{deg} \sup \left\{D, D^{\prime}\right\}=\operatorname{dim}\left|\sup \left\{D, D^{\prime}\right\}\right| .
\end{aligned}
$$

The lemma follows.

Lemma 9.4. Let $x, y \in K \backslash \mathbb{k}$ and let $a=\operatorname{deg}(x)_{0}=\operatorname{deg}(x)_{\infty}$ and $b=\operatorname{deg}(y)_{0}=\operatorname{deg}(y)_{\infty}$. If $(x)$ and $(y)$ have disjoint supports and $\operatorname{deg} D>a+b$ then

$$
x|D|+y|D|=\left|D+(x)_{\infty}+(y)_{\infty}\right|
$$

Proof. By (9.2) we have $x|D|=|D-(x)|, y|D|=|D-(y)|$. We have inf $\{D-(x), D-(y)\}=D-(x)_{0}-(y)_{0}$ by hypothesis and so $\operatorname{deg} \inf \{D-(x), D-(y)\}=\operatorname{deg} D-a-b>0$. Thus by Lemma 9.3 we have

$$
x|D|+y|D|=|D-(x)|+|D-(y)|=\left|\sup _{37}\{D-(x), D-(y)\}\right|=\left|D+(x)_{\infty}+(y)_{\infty}\right|,
$$


again using our assumption on the supports of $(x)$ and $(y)$.

In the next result, let $\sigma$ be an infinite order translation automorphism of $E$. We work inside the Ore extension $K[t ; \sigma]$.

Lemma 9.5. Let $y \in K \backslash \mathbb{k}$ be such that all points in the support of $(y)$ have disjoint $\sigma$-orbits, and let $d:=\operatorname{deg}(y)_{0}$. Let $D$ be a divisor with $\operatorname{deg} D>2 d$. Let $V:=|D| \cdot t$ and let $W:=\mathbb{k}+\mathbb{k} y+V$. If $n \geqslant m \geqslant 1$, then $W^{n} \cap K t^{m}=|F(n, m)| t^{m}$, where

$$
F(n, m):=D+\sigma^{-1} D+\cdots+\sigma^{-m+1} D+(n-m)\left((y)_{\infty}+\left(y^{\sigma}\right)_{\infty}+\cdots+\left(y^{\sigma^{m}}\right)_{\infty}\right) .
$$

Proof. Certainly $W^{n} \cap K t^{m}$ is spanned by

$$
\left\{y^{i_{0}} V\left(y^{\sigma}\right)^{i_{1}} V^{\sigma} \cdots V^{\sigma^{m-1}}\left(y^{\sigma^{m}}\right)^{i_{m}} t^{m}: \sum i_{j} \leqslant n-m, i_{j} \geqslant 0 \forall j\right\} .
$$

Each of these is contained $|F(n, m)| t^{m}$, giving one inclusion.

We show the other inclusion by induction on $n \geqslant m$. Certainly $W^{m} \cap K t^{m}=V^{m}=|F(m, m)| t^{m}$, so assume that $n>m$. By induction, $W^{n-1} \cap K t^{m}=|F| t^{m}$, where $F=F(n-1, m)$.

Choose $0 \leqslant j \leqslant m-1$. Then

$$
\begin{aligned}
W^{n} \cap K t^{m} & \supseteq\left(y^{\sigma^{j}} W^{n-1}+y^{\sigma^{j+1}} W^{n-1}\right) \cap K t^{m}=\left(y^{\sigma^{j}}|F|+y^{\sigma^{j+1}}|F|\right) t^{m} \\
& =\left|F+\left(y^{\sigma^{j}}\right)_{\infty}+\left(y^{\sigma^{j+1}}\right)_{\infty}\right| t^{m}, \quad \text { by Lemma 9.4. }
\end{aligned}
$$

where we have used that $\operatorname{deg} F \geqslant \operatorname{deg} D>2 d$.

So

$$
\begin{aligned}
W^{n} \cap K t^{m} & \supseteq \sum_{j=0}^{m-1}\left|F+\left(y^{\sigma^{j}}\right)_{\infty}+\left(y^{\sigma^{j+1}}\right)_{\infty}\right| t^{m}=\left|\sup _{j} F+\left(y^{\sigma^{j}}\right)_{\infty}+\left(y^{\sigma^{j+1}}\right)_{\infty}\right| t^{m} \quad \text { using Lemma } 9.3 \\
& =\left|F+\sum_{j=0}^{m}\left(y^{\sigma^{j}}\right)_{\infty}\right| t^{m} \quad \text { by assumption on } y \\
& =|F(n, m)| t^{m}
\end{aligned}
$$

as needed.

We now give a result on the GK-dimension of overrings of TCRs of elliptic curves.

Proposition 9.6. Let $B:=B(E, \mathcal{L}, \sigma)$ where $E$ is an elliptic curve, $\sigma$ has infinite order, and $\mathcal{L}$ is an ample invertible sheaf on $E$, and let $B \varsubsetneqq C \subseteq Q_{\text {gr }}(B)$ where $C$ is $\mathbb{Z}$-graded with $\operatorname{dim} C_{0}>1$. Then GKdim $C \geqslant 3$.

Proof. We write $K:=\mathbb{k}(E)$ and $C=\bigoplus C_{i} t^{i} \subseteq K\left[t, t^{-1} ; \sigma\right]$. By hypothesis there exists $y \in C_{0} \backslash \mathbb{k}$. Let $d:=\operatorname{deg}(y)_{0}>0$.

Choose a positive integer $c$ so that all points in the support of $(y)$ have disjoint $\sigma^{c}$-orbits and so that $\operatorname{deg} \mathcal{L}_{c}>2 d$. Let $C^{\prime}:=(B\langle y\rangle)^{(c)}$. It suffices to prove that $\operatorname{GKdim} C^{\prime} \geqslant 3$. 
We note that $C^{\prime}=\mathbb{k}\langle W\rangle$ where $W:=\mathbb{k}+\mathbb{k} y+H^{0}\left(E, \mathcal{L}_{c}\right) t^{c}$. By Lemma 9.5 we have

$$
\begin{aligned}
\operatorname{dim} W^{n} & \geqslant \sum_{m=1}^{n} \operatorname{dim}\left(W^{n} \cap K t^{m c}\right) \geqslant \sum_{m=1}^{n}(n-m) m=n \sum_{m=1}^{n} m-\sum_{m=1}^{n} m^{2} \\
& =n\left(\begin{array}{c}
n+1 \\
2
\end{array}\right)-\frac{n(n+1)(2 n+1)}{6}=\frac{n^{3}}{6}+O\left(n^{2}\right),
\end{aligned}
$$

and so $\operatorname{GKdim} C^{\prime} \geqslant 3$. (A little more work will show that $\operatorname{GKdim} C^{\prime}=3$ but this is all that is required.)

Applying this to minimal elliptic surfaces, we obtain:

Corollary 9.7. Let $T$ be a minimal elliptic surface and let $T \varsubsetneqq R \subset T_{(g)}$ be a noetherian graded overring of $T$. Then GKdim $R \geqslant 4$. In particular, by Theorem [6.16, this holds for $T=Q_{V d B}^{(2)}$ or $S k l^{(3)}$ or $S k l^{(4)}$.

Proof. By definition, $R$ cannot be cg. Note that one cannot have $R_{\leqslant 0}=\mathbb{k}$, as this would contradict Remark 2.2(2). Thus Remark 2.2(1) implies that $R_{0} \supsetneq \mathbb{k}$.

So, suppose that $R_{0} \ni x_{1}^{-1} x_{2} g^{m}$, for some $m \geqslant 0$ and $x_{i} \in T \backslash g T$. Then $\widehat{R}_{\leqslant 0} \ni z=x_{1}^{-1} x_{2}$ with $z \in$ $T_{(g)} \backslash g T_{(g)}$. Thus $\widehat{R}+g T_{(g)} \supseteq T\langle z\rangle$ and hence $\bar{R} \supseteq \bar{T}\langle\bar{z}\rangle$. Therefore, by Proposition9.6 GKdim $\widehat{R} / g \widehat{R} \geqslant 3$.

Note that $R^{\circ}=(\widehat{R})^{\circ}$. Therefore, by [RSS1, Lemmas 2.1 and 2.2], there is a filtered isomorphism $\theta$ : $R^{\circ} \stackrel{\sim}{\longrightarrow} R /(g-1) R$, with $\operatorname{gr}\left(R^{\circ}\right)=\widehat{R} / g \widehat{R}$. Thus, GKdim $\operatorname{gr}\left(R^{\circ}\right) \geqslant 3$. By [KL, Lemma 6.5], GKdim $R^{\circ} \geqslant$ $\operatorname{GKdim} \operatorname{gr}\left(R^{\circ}\right)$. Therefore, by [KL, Proposition 3.15], GKdim $R \geqslant \operatorname{GKdim} R^{\circ}+1$. Putting this together gives $\operatorname{GKdim} R \geqslant 4$.

We also have:

Theorem 9.8. Let $T=S k l^{(3)}$ or $T=\left(S k l^{\prime}\right)^{(4)}$ or $T=Q_{V d B}{ }^{(2)}$. If $A$ is an algebra with $T^{\circ} \varsubsetneqq A \subseteq Q\left(T^{\circ}\right)$ then $\operatorname{GKdim} A \geqslant 3=\mathrm{GKdim} T^{\circ}+1$.

Proof. We first establish the result if $T^{\circ}$ is simple. Using Notation 4.7 and [RSS2, p.2099], $A=(\Phi A)^{\circ}$ and so $T \varsubsetneqq \Phi A \subseteq T_{(g)}$. Since $\Phi A=\widehat{\Phi A}$, Remark 6.17 implies that $\operatorname{dim}(\overline{\Phi A})_{0}>1$. Thus by Proposition 9.6 , $\operatorname{GKdim} \overline{\Phi A} \geqslant 3$.

Now suppose that $\operatorname{GKdim} A:=\alpha<3$. Since $\Phi(A) \subseteq A\left[g, g^{-1}\right]$, it follows from [KL, Lemma 3.1 and Proposition 3.5] that $\beta:=\operatorname{GKdim} \Phi(A) \leqslant \operatorname{GKdim} A\left[g, g^{-1}\right] \leqslant \alpha+1$. Now, as $g T_{(g)} \cap \Phi(A)=g \Phi(A)$ is a nonzero ideal of the domain $\Phi A$, it follows from [KL, Proposition 3.15] that GKdim $\overline{\Phi A} \leqslant \beta-1 \leqslant \alpha<3$. This contradicts the first paragraph of the proof.

Now suppose that $T^{\circ}$ is not simple. As in the proof of Theorem6.16, there are a simple elliptic algebra $T_{\infty}$ and a $\left(T_{\infty}, T\right)$-bimodule $K$, which is finitely generated on both sides by Corollary 6.11 so that $\operatorname{End}_{T}(K)=$ $T_{\infty}$ and $\operatorname{End}_{T_{\infty}}(K)=T$. Set $\mathcal{K}:=K^{\circ}$; thus $\mathcal{L}:=\mathcal{K}^{*} \mathcal{K} \subset T^{\circ}$ and $\mathcal{K} \mathcal{K}^{*}=T_{\infty}^{\circ}$ as $T_{\infty}^{\circ}$ is simple. Let $C:=T_{\infty}^{\circ}+\mathcal{K} A \mathcal{K}^{*}$. If $C=T_{\infty}^{\circ}$, then $\mathcal{L} A \mathcal{L} \subseteq \mathcal{K}^{*} T_{\infty}^{\circ} \mathcal{K} \subseteq T^{\circ}$. As $T^{\circ}$ is a maximal order by Remark 6.8 , this implies that $A \subseteq T^{\circ}$, a contradiction. Thus $C \supsetneqq T_{\infty}^{\circ}$ and so, by the first part of the proof, GKdim $C \geqslant 3$. 
Let $A^{\prime}:=T^{\circ}+\mathcal{K}^{*} C \mathcal{K} \subseteq A$. Now, $\mathcal{K} A^{\prime}=\mathcal{K}+\mathcal{K} \mathcal{K}^{*} C \mathcal{K}=C \mathcal{K}$, and so $C \mathcal{K}$ is a $\left(C, A^{\prime}\right)$-bimodule which is finitely generated, and clearly torsionfree, on both sides. Thus by [KL, Corollary 5.4],

$$
3 \leqslant \operatorname{GKdim} C=\operatorname{GKdim}_{C}(C \mathcal{K})=\operatorname{GKdim}(C \mathcal{K})_{A^{\prime}}=\operatorname{GKdim} A^{\prime} \leqslant \operatorname{GKdim} A
$$

giving the result.

We conjecture that Theorem 9.8 holds if $T$ is any minimal elliptic surface.

Remark 9.9. Theorem 9.8 is reminiscent of a striking result of Makar-Limanov ML on the localised Weyl algebra. If char $\mathbb{k}=0$ and $A:=\mathbb{k}(x)\left[\partial, \partial^{-1}\right]$, for $\partial:=\frac{d}{d x}$ he shows that if $B$ is a ring with $A \varsubsetneqq B \subseteq \operatorname{Fr}(A)$ then $\operatorname{GKdim} B>\operatorname{GKdim} A$; in fact $\operatorname{GKdim} B=\infty$.

\section{Appendix A. Commutative algebras}

We end this paper with a few comments on the commutative analogues of the results in this paper; thereby justifying some of the comments from the introduction by noting that elliptic algebras are noncommutative versions of anticanonical (homogeneous coordinate) rings of del Pezzo surfaces, and by exploring some of the properties of these rings. This result will not be used in the body of the paper, so can be skipped on first reading.

Lemma A.1. Let $T$ be a cg commutative domain that is generated by $T_{1}$ and so that there is $g \in T_{1}$ with $T / g T$ isomorphic to the homogeneous coordinate ring $B:=B(E, \mathcal{L})$ of an elliptic curve $E$ with respect to an ample line bundle $\mathcal{L}$ on $E$. Suppose also that $X:=\operatorname{Proj} T$ is nonsingular. Then $X$ is a del Pezzo surface of degree $\geqslant 3$, and $T$ is isomorphic to the anticanonical coordinate ring of $X$.

Proof. We need to prove that $\omega_{X}^{-1}$ is ample.. We use $C \cdot C^{\prime}$ to denote the intersection product on $X$. From the setup, $g$ defines the elliptic curve $E \subset X$, and $\mathcal{O}_{X}(E) \cong \mathcal{O}_{X}(1)$ with $\left.\mathcal{L} \cong \mathcal{O}_{X}(E)\right|_{E}$. Since $B(E, \mathcal{L})$ is generated in degree $1, d:=\operatorname{deg} \mathcal{L}=E^{2}$, where $E^{2}:=E \cdot E \geqslant 3$. Letting $K=K_{X}$ be the canonical divisor on $X$, by adjunction [Ha, Proposition V.1.5] we have

$$
0=E \cdot(E+K)
$$

For a sheaf $\mathcal{M}$ on $X$, let $h^{i}(X, \mathcal{M})=\operatorname{dim} H^{i}(X, \mathcal{M})$. Then we have

$$
\begin{aligned}
1+d \frac{n(n+1)}{2} & =\sum_{k=0}^{n} \operatorname{dim} B_{k}=\operatorname{dim} T_{n} & & \text { as } B=T / g T \\
& =h^{0}\left(X, \mathcal{O}_{X}(n E)\right)=\chi\left(\mathcal{O}_{X}(n E)\right) & & \text { for } n \gg 0 \\
& =\chi\left(\mathcal{O}_{X}\right)+\frac{1}{2} n E \cdot(n E-K) & & \text { by Riemann-Roch } \\
& =\chi\left(\mathcal{O}_{X}\right)+\frac{n(n+1)}{2} E^{2} & & \text { by A.2. }
\end{aligned}
$$

Since $E^{2}=d, \chi\left(\mathcal{O}_{X}\right)=1$. 
Further, note that $K$ is not effective, as $E$ is ample and $K \cdot E=-E^{2}<0$. In particular, $h^{2}\left(X, \mathcal{O}_{X}\right)=$ $h^{0}(X, K)=0$, and as $1=\chi\left(\mathcal{O}_{X}\right)=h^{0}\left(X, \mathcal{O}_{X}\right)$, we have $h^{1}\left(X, \mathcal{O}_{X}\right)=0$.

Consider the exact sequence

$$
H^{1}\left(X, \mathcal{O}_{X}\right) \longrightarrow H^{1}\left(X, \mathcal{O}_{E}\right) \longrightarrow H^{2}\left(X, \mathcal{O}_{X}(-E)\right) \longrightarrow H^{2}\left(X, \mathcal{O}_{X}\right) .
$$

Since the outside terms are zero, it follows that that $\mathbb{k} \cong H^{2}\left(X, \mathcal{O}_{X}(-E)\right) \cong H^{0}\left(X, \mathcal{O}_{X}(K+E)\right)$. Thus $K+E$ is effective. By (A.2) and the fact that $E$ is ample, $K+E=0$, so $\omega_{X}^{-1} \cong \mathcal{O}_{X}(E)$. In particular, $\omega_{X}^{-1}$ is ample. Thus, by definition, $X$ is del Pezzo, while $T$ and $B\left(X, \omega_{X}^{-1}\right)=B\left(X, \mathcal{O}_{X}(E)\right)$ are equal in large degree. But the Hilbert series of $B\left(X, \omega_{X}^{-1}\right)$ is the same as that of $T$, which was calculated above, by [Ko, Corollary III.3.2.5]. Thus $T=B\left(X, \omega_{X}^{-1}\right)$ is the anticanonical coordinate ring of $X$.

Remark A.3. Line modules of self-intersection $(-1)$ play a crucial role in the noncommutative theory we are developing. We remark that in the context of Lemma A.1 all line modules correspond to lines of selfintersection (-1). Indeed, let $X$ be a smooth del Pezzo surface of degree $\geqslant 3$, and let $T:=B\left(X, \omega_{X}^{-1}\right)$ be the anticanonical ring. Let $L$ be a line module over $T$. Then there is a curve $C$ on $X$ so that, for $n$ sufficiently large, $L_{n}=H^{0}\left(C,\left.\omega_{X}^{-n}\right|_{C}\right)$, by Serre's Theorem [Ha, Exercise II.5.9]. From the Hilbert series of $L$ and Riemann-Roch on $C$, we obtain that $-K_{X} \cdot C=1$ and that $\chi\left(\mathcal{O}_{C}\right)=1$. Thus by [Ko, Lemma III.3.6.1] $C$ is a smooth rational curve of self-intersection -1 .

It may seem counterintuitive that blowing up a point corresponds to constructing a subalgebra, but it can be quite natural in the commutative case, as the following example illustrates.

Remark A.4. Let $X$ be the blowup of $\mathbb{P}^{2}$ at $p$. Then the anticanonical ring of $X$ is the subalgebra of $\mathbb{k}[x, y, z]^{(3)}$ generated by 3 -forms vanishing at $p$.

Proof. To see this, let $\pi: X \rightarrow \mathbb{P}^{2}$ be the blowdown morphism and let $L$ be the exceptional line. By [Ha, Proposition V.3.3], $-K_{X}=\pi^{*}\left(-K_{\mathbb{P}^{2}}\right)-L$. Thus

$$
\begin{aligned}
H^{0}\left(X, \omega_{X}^{-1}\right)=H^{0}\left(X,-K_{X}\right) & =\left\{f \in H^{0}\left(X, \pi^{*}\left(-K_{\mathbb{P}^{2}}\right)\right):\left.f\right|_{L} \equiv 0\right\} \\
& =\left\{f \in H^{0}\left(\mathbb{P}^{2},-K_{\mathbb{P}^{2}}\right): f(p)=0\right\} .
\end{aligned}
$$

As the anticanonical ring of a degree 8 del Pezzo surface is generated in degree 1 , this is sufficient.

\section{INDEX OF NOTATION}

Auslander-Gorenstein algebra

associated elliptic curve

birational algebras

Cohen-Macaulay (CM) ring

$\mathrm{CM}$ and MCM modules

connected graded (cg) algebra critical and pure modules

degree of an elliptic algebra

$E^{11}(M)=\operatorname{Ext}_{T}^{1}\left(\operatorname{Ext}_{T}^{1}(M, T), T\right)$

elliptic algebra $T$

finitely graded algebra

GKdim $M$ Gelfand-Kirillov dimension
7 
$g$-torsion and Goldie torsion modules

$g$-divisible module, $g$-divisible hull $\hat{X}$

Hilbert series hilb $(M)$

$j(M)$ homological grade of a module

linear, line and point modules

locally hereditary algebra

locally simple algebra

$M^{\circ}, T^{\circ}$ localisations

maximal order

minimal elliptic surface

multiplicity of $M$

\begin{tabular}{|c|c|}
\hline 7 & $\Phi \mathcal{M}:=\bigoplus_{n \in \mathbb{Z}}(\Phi \mathcal{M})_{n}$ \\
\hline 11 & quadric elliptic algebra $T=Q_{V d B}^{(2)}$ \\
\hline 7 & gr- $R$, qgr- $R$ (quotient) module category \\
\hline 7 & $Q_{g r}(R)$ graded quotient ring \\
\hline 7 & $T_{\infty}$ special quadric elliptic algebra \\
\hline 32 & singularity category, $A_{1}$ singularity \\
\hline 14 & Sklyanin algebra, $S=S k l(a, b, c)$ \\
\hline 5 & Sklyanin elliptic algebra, $T=S^{(3)}$ \\
\hline 5 & smooth qgr- $T$ \\
\hline 4 & TCR twisted coordinate ring $B(X, \mathcal{L}, \theta)$ \\
\hline 22 & Van den Bergh quadric $Q_{V d B}=Q_{V d B}(r)$ \\
\hline
\end{tabular}

\section{REFERENCES}

[Aj] K. Ajitabh, Residue complex for Sklyanin algebras of dimension three, Adv. Math. 144 (1999), no. 2, $137-160$.

[Ar] M. Artin, Some problems on three-dimensional graded domains, in: Representation Theory and Algebraic Geometry (Waltham, MA, 1995), 1-19, London Math. Soc. Lecture Note Ser., no. 238, Cambridge Univ. Press, Cambridge, 1997.

[ASc] M. Artin and W. F. Schelter, Graded algebras of global dimension 3, Adv. in Math. 66 (1987), no. 2, $171-216$.

[AS] M. Artin and J. T. Stafford, Noncommutative graded domains with quadratic growth, Invent. Math. 122 (1995), 231-276.

[ATV1] M. Artin, J. Tate, and M. Van den Bergh, Some algebras associated to automorphisms of elliptic curves, The Grothendieck Festschrift Vol. I, Progr. Math., Vol. 86, Birkhäuser Boston, Boston, 1990, pp. 33-85.

[ATV2] M. Artin, J. Tate, and M. Van den Bergh, Modules over regular algebras of dimension 3, Invent. Math. 106 (1991), 335-388.

[AV] M. Artin and M. Van den Bergh, Twisted homogeneous coordinate rings J. Algebra 133 (1990), 249-271.

[AB] M. Auslander and M. Bridger, Stable module theory, Mem. Amer. Math. Soc. No. 94, 1969.

[BW] Y. Berest, and G. Wilson, Ideal classes of the Weyl algebra and noncommutative projective geometry, Int. Math. Res. Not. 26 (2002), 1347-1396.

[Bj] J.-E. Björk, Filtered Noetherian rings, in Noetherian Rings and Their Applications, (ed: L. W. Small) Math. Surveys and Monographs, No. 24, pp. 59-98, Amer. Math. Soc, Providence, RI, 1987.

[Bu] R.-O. Buchweitz, Maximal Cohen-Macaulay modules and Tate-cohomology over Gorenstein rings, unpublished manuscript (1986), see http://hdl.handle.net/1807/16682

$[\mathrm{BH}] \quad$ L. D. Bush Hipwood, Maximal orders in the Sklyanin algebra, Ph.D. thesis, University of Manchester, 2018. See also arXiv:1812.04137.

[Cr] S. Crawford, Singularities of noncommutative surfaces, Ph.D. thesis, University of Edinburgh, 2018. Available at https://era.ed.ac.uk/handle/1842/31543.

[DL] K. De Laet, On the center of 3-dimensional and 4-dimensional Sklyanin algebras, J. Algebra 487 (2017), $244 ? 268$.

[Go] K. R. Goodearl, Localization and splitting in hereditary noetherian prime rings, Pacific J. Math. 53 (1974), $137-151$.

[Ha] R. Hartshorne, Algebraic geometry, Springer-Verlag, Berlin, 2006.

[Sh] V. A. Iskovskikh and I. R. Shafarevich, Algebraic surfaces. Algebraic geometry, II, 127-262, Encyclopaedia Math. Sci., 35, Springer, Berlin, 1996.

[KRS] D. S. Keeler, D. Rogalski and J. T. Stafford, Naive noncommutative blowing up, Duke Math. J. 126 (2003), $491-546$.

[Ko] J. Kollár, Rational curves on algebraic varieties. Ergebnisse der Mathematik und ihrer Grenzgebiete. 3. Folge. A Series of Modern Surveys in Mathematics, 32, Springer-Verlag, Berlin, 1996. 
[KL] G. R. Krause and T. H. Lenagan, Growth of Algebras and Gelfand-Kirillov Dimension, Research Notes in Math. Vol. 116, Pitman Boston, 1985.

$[\mathrm{Ku}] \quad$ J. Kuzmanovich, Localizations of Dedekind prime rings, J. Algebra 21 (1972), 378-393.

[Le] T. Levasseur, Some properties of noncommutative regular graded rings, Glasgow Math. J. 34 (1992), 277-300.

[LS] T. Levasseur and S. P. Smith, Modules over the 4-dimensional Sklyanin algebra, Bull. Soc. Math. France 121 (1993), 35-90.

[ML] L. Makar-Limanov, On subalgebras of the first Weyl skewfield, Comm. Algebra 19 (1991), 1971-1982.

[MS] I. Mori and S. P. Smith, The Grothendieck group of a quantum projective space bundle, K-Theory 37 (2006), $263-289$.

[NS] T. Nevins and J. T. Stafford, Sklyanin algebras and Hilbert schemes of points, Adv. Math. 210 (2007), 405-478.

[Rg] D. Rogalski, Blowup subalgebras of the Sklyanin algebra, Adv. Math. 226 (2011), 1433-1473.

[RSS1] D. Rogalski, S. J. Sierra and J. T. Stafford, Algebras in which every subalgebra is noetherian, Proc. Amer. Math. Soc. 142 (2014), 2983-2990.

[RSS2] D. Rogalski, S. J. Sierra and J. T. Stafford, Classifying orders in the Sklyanin algebra, Algebra and Number Theory 9 (2015), 2055-2119.

[RSS3] D. Rogalski, S. J. Sierra and J. T. Stafford, Noncommutative blowups of elliptic algebras, Algebra and Rep. Theory 18 (2015), 491-529.

[RSS4] D. Rogalski, S. J. Sierra and J. T. Stafford, Ring-theoretic blowing down: I, J. Noncomm. Geom., 11 (2017), $1465-1520$.

[RS] D. Rogalski and J. T. Stafford, A class of noncommutative projective surfaces, Proc. London Math. Soc., 99 (2009), $100-144$.

[Sc] A. H. Schofield, Stratiform simple Artinian rings, Proc. London Math. Soc. 53 (1986), 267-287.

[Si] S. J. Sierra, Classifying birationally commutative projective surfaces, Proc. London Math. Soc. 103 (2011), 139-196.

[SW] L. W. Small and R. B. Warfield, Jr., Prime affine algebras of Gelfand-Kirillov dimension one J. Algebra 91 (1984), 386-389.

[SSf] S. P. Smith and J. T. Stafford, Regularity of the 4-dimensional Sklyanin algebra, Compositio Math. 83 (1992), $259-289$.

[SSn] S. P. Smith and J. M. Staniszkis, Irreducible representations of the 4-dimensional Sklyanin algebra at points of infinite order, J. Algebra 160 (1993), 57-86.

[SmV] S. P. Smith and M Van den Bergh, Non-commutative quadric surfaces, J. Noncomm. Geom. 7 (2013), 817-856.

[StV] J. T. Stafford and M Van den Bergh, Noncommutative projective curves and surfaces, Bull. Amer. Math. Soc. 38 (2001), 171-216.

[VB1] M. Van den Bergh, A translation principle for the four-dimensional Sklyanin algebras, J. Algebra 184 (1996), 435-490.

[VB2] M. Van den Bergh, Blowing up of non-commutative smooth surfaces, Mem. Amer. Math. Soc. 154 (2001), no. 734.

[VB3] M. Van den Bergh, Noncommutative quadrics, Int. Math. Res. Not. IMRN (2011), no. 17, 398-4026.

(Rogalski) Department of Mathematics, UCSD, La Jolla, CA 92093-0112, USA.

E-mail address: drogalski@ucsd.edu

(Sierra) School of Mathematics, University of Edinburgh, Edinburgh EH9 3FD, Scotland.

E-mail address: s.sierra@ed.ac.uk

(Stafford) School of Mathematics, The University of Manchester, Manchester M13 9PL, England.

E-mail address: Toby.Stafford@manchester.ac.uk 